\title{
ON THE BIFURCATION SET OF UNIQUE EXPANSIONS
}

\author{
CHARLENE KALLE, DERONG KONG, WENXIA LI, AND FAN LÜ
}

\begin{abstract}
Given a positive integer $M$, for $q \in(1, M+1]$ let $\mathcal{U}_{q}$ be the set of $x \in[0, M /(q-$ 1)] having a unique $q$-expansion with the digit set $\{0,1, \ldots, M\}$, and let $\mathbf{U}_{q}$ be the set of corresponding $q$-expansions. Recently, Komornik et al. showed in [23] that the topological entropy function $H: q \mapsto h_{t o p}\left(\mathbf{U}_{q}\right)$ is a Devil's staircase in $(1, M+1]$.

Let $\mathscr{B}$ be the bifurcation set of $H$ defined by
\end{abstract}

$$
\mathscr{B}=\{q \in(1, M+1]: H(p) \neq H(q) \quad \text { for any } \quad p \neq q\} .
$$

In this paper we analyze the fractal properties of $\mathscr{B}$, and show that for any $q \in \mathscr{B}$,

$$
\lim _{\delta \rightarrow 0} \operatorname{dim}_{H}(\mathscr{B} \cap(q-\delta, q+\delta))=\operatorname{dim}_{H} \mathcal{U}_{q},
$$

where $\operatorname{dim}_{H}$ denotes the Hausdorff dimension. Moreover, when $q \in \mathscr{B}$ the univoque set $\mathcal{U}_{q}$ is dimensionally homogeneous, i.e., $\operatorname{dim}_{H}\left(\mathcal{U}_{q} \cap V\right)=\operatorname{dim}_{H} \mathcal{U}_{q}$ for any open set $V$ that intersect $\mathcal{U}_{q}$.

As an application we obtain a dimensional spectrum result for the set $\mathscr{U}$ containing all bases $q \in(1, M+1]$ such that 1 admits a unique $q$-expansion. In particular, we prove that for any $t>1$ we have

$$
\operatorname{dim}_{H}(\mathscr{U} \cap(1, t])=\max _{q \leq t} \operatorname{dim}_{H} \mathcal{U}_{q} .
$$

We also consider the variations of the sets $\mathscr{U}=\mathscr{U}(M)$ when $M$ changes.

\section{INTRODUCTION}

Fix a positive integer $M$. For any $q \in(1, M+1]$ each $x \in I_{q, M}:=[0, M /(q-1)]$ has a $q$-expansion, i.e., there exists a sequence $\left(x_{i}\right)=x_{1} x_{2} \ldots$ with each $x_{i} \in\{0,1, \ldots, M\}$ such that

$$
x=\sum_{i=1}^{\infty} \frac{x_{i}}{q^{i}}=: \pi_{q}\left(\left(x_{i}\right)\right) .
$$

The sequence $\left(x_{i}\right)$ is called a $q$-expansion of $x$. If no confusion arises the alphabet is always assumed to be $\{0,1, \ldots, M\}$.

Non-integer base expansions have received a lot of attention since the pioneering papers of Rényi [34] and Parry [33]. It is well known that for any $q \in(1, M+1)$ Lebesgue almost every $x \in I_{q, M}$ has a continuum of $q$-expansions (cf. [35, 11]). Moreover, for any $k \in \mathbb{N} \cup\left\{\aleph_{0}\right\}$

2010 Mathematics Subject Classification. Primary:11A63; Secondary: 37B10, 28A78.

Key words and phrases. Bifurcation set, topological entropy, univoque sets, univoque bases, Hausdorff dimensions, Devil's staircase. 
there exist $q \in(1, M+1]$ and $x \in I_{q, M}$ such that $x$ has precisely $k$ different $q$-expansions (see e.g., [19, 37]). For more information on non-integer base expansions we refer the reader to the survey paper [22] and the references therein.

In this paper we focus on studying unique $q$-expansions. For $q \in(1, M+1]$ let

$$
\mathcal{U}_{q}:=\left\{x \in I_{q, M}: x \text { has a unique } q \text {-expansion }\right\},
$$

and let $\mathbf{U}_{q}=\pi_{q}^{-1}\left(\mathcal{U}_{q}\right)$ be the set of corresponding $q$-expansions. These sets have been the object of study in many articles and have a very rich topological structure (see for example [25, 15]). Komornik et al. studied in [23] the Hausdorff dimension of $\mathcal{U}_{q}$, and showed that the dimension function $D: q \mapsto \operatorname{dim}_{H} \mathcal{U}_{q}$ has a Devil's staircase behavior (see also [3]). Moreover, they showed that the entropy function

$$
H:(1, M+1] \rightarrow[0, \log (M+1)] ; \quad q \mapsto h_{t o p}\left(\mathbf{U}_{q}\right)
$$

is a Devil's staircase (see Lemma 2.4 below). Recently, Alcaraz Barrera et al. investigated in [1] the dynamical properties of $\mathcal{U}_{q}$, and determined the maximal intervals on which the entropy function $H$ is constant.

Let $\mathscr{B}$ be the bifurcation set of the function $H$ defined by

$$
\mathscr{B}=\{q \in(1, M+1]: H(p) \neq H(q) \text { for any } p \neq q\} .
$$

Then $\mathscr{B}$ is the set of bases where the entropy function $H$ is not locally constant. In [1] Alcaraz Barrera et al. gave a characterization of $\mathscr{B}$ and showed that $\mathscr{B}$ has full Hausdorff dimension. In particular, we have

$$
\mathscr{B}=\left(q_{K L}, M+1\right] \backslash \bigcup\left[p_{L}, p_{R}\right],
$$

where $q_{K L}$ is the Komornik-Loreti constant (cf. [24]) and the union on the right hand side is countable and pairwise disjoint (see Section 2 below for more explanation).

From [15] we know that the univoque set $\mathcal{U}_{q}$ has a fractal structure and might have isolated points. Our first result states that for $q \in \mathscr{B}$ the univoque set $\mathcal{U}_{q}$ is dimensionally homogeneous, i.e., the local Hausdorff dimension of $\mathcal{U}_{q}$ equals the full dimension of $\mathcal{U}_{q}$.

Theorem 1. Let $q \in\left(q_{K L}, M+1\right] \backslash \bigcup\left(p_{L}, p_{R}\right]$. Then for any open set $V \subseteq \mathbb{R}$ with $\mathcal{U}_{q} \cap V \neq \emptyset$ we have

$$
\operatorname{dim}_{H}\left(\mathcal{U}_{q} \cap V\right)=\operatorname{dim}_{H} \mathcal{U}_{q}
$$

Remark 1.1.

(1) Note by (1.2) that $\mathscr{B} \subset\left(q_{K L}, M+1\right] \backslash \bigcup\left(p_{L}, p_{R}\right]$. So Theorem 11 implies that the univoque set $\mathcal{U}_{q}$ is dimensionally homogeneous for any $q \in \mathscr{B}$. 
(2) In Theorem 3.6 we give a complete characterization of the set

$$
\left\{q \in(1, M+1]: \mathcal{U}_{q} \text { is dimensionally homogeneous }\right\} \text {. }
$$

It turns out that the Lebesgue measure of this set is positive and strictly smaller than $M$.

Throughout the paper we will use $\bar{A}$ to denote the topological closure of a set $A \subset \mathbb{R}$. Our second result presents a close relationship between the bifurcation set $\overline{\mathscr{B}}$ and the univoque $\operatorname{sets} \mathcal{U}_{q}$.

Theorem 2. For any $q \in \overline{\mathscr{B}}$ we have

$$
\lim _{\delta \rightarrow 0} \operatorname{dim}_{H}(\overline{\mathscr{B}} \cap(q-\delta, q+\delta))=\operatorname{dim}_{H} \mathcal{U}_{q}
$$

Remark 1.2.

(1) Since by (1.2) and (2.5) the difference between $\mathscr{B}$ and $\overline{\mathscr{B}}$ is countable, Theorem 2 also holds if we replace $\overline{\mathscr{B}}$ by $\mathscr{B}$.

(2) Note that $\operatorname{dim}_{H} \mathcal{U}_{q}>0$ for any $q>q_{K L}$ (see Lemma 2.4 below). As a consequence of Theorem 2 it follows that

$$
q \in \overline{\mathscr{B}} \backslash\left\{q_{K L}\right\} \quad \Longleftrightarrow \quad \lim _{\delta \rightarrow 0} \operatorname{dim}_{H}(\overline{\mathscr{B}} \cap(q-\delta, q+\delta))=\operatorname{dim}_{H} \mathcal{U}_{q}>0 .
$$

Recently, Allaart et al. [2, Corollary 3] gave another characterization of $\overline{\mathscr{B}}$, and showed that

$$
q \in \overline{\mathscr{B}} \backslash\left\{q_{K L}\right\} \quad \Longleftrightarrow \quad \lim _{\delta \rightarrow 0} \operatorname{dim}_{H}(\mathscr{U} \cap(q-\delta, q+\delta))=\operatorname{dim}_{H} \mathcal{U}_{q}>0,
$$

where $\mathscr{U}:=\left\{q \in(1, M+1]: 1 \in \mathcal{U}_{q}\right\}$.

It is well-known that the univoque set $\mathcal{U}_{q}$ has a close connection with the set $\mathscr{U}=\mathscr{U}(M)$ of univoque bases $q \in(1, M+1]$ for which 1 has a unique $q$-expansion with alphabet $\{0,1, \ldots, M\}$. For example, in [15] De Vries and Komornik showed that $\mathcal{U}_{q}$ is closed if and only if $q \notin \overline{\mathscr{U}}$. The set $\mathscr{U}$ has many interesting properties itself. Erdös et al. showed in [18] that $\mathscr{U}$ is an uncountable set of zero Lebesgue measure. Daróczy and Kátai proved in [14] that the Hausdorff dimension of $\mathscr{U}$ is 1 (see also [23]). Komornik and Loreti showed in 24] that the smallest element of $\mathscr{U}$ is $q_{K L}$. In [25] the same authors studied the topological properties of $\mathscr{U}$, and showed that its closure $\overline{\mathscr{U}}$ is a Cantor set. Recently, Kong et al. proved in [28] that for any $q \in \overline{\mathscr{U}}$ we have

$$
\operatorname{dim}_{H}(\overline{\mathscr{U}} \cap(q-\delta, q+\delta))>0 \quad \text { for any } \delta>0 .
$$

On a different note, in [9] Bonanno et al. introduced a set

$$
\Lambda=\left\{x \in[0,1]: S^{k} x \leq x \text { for all } n \geq 0\right\},
$$


where $S$ is the tent map defined by $S: x \mapsto \min \{2 x, 2-2 x\}$ and showed that there is a one to one correspondence between the set $\mathscr{U}(1)$ and the set $\Lambda \backslash \mathbb{Q}_{1}$, where $\mathbb{Q}_{1}$ is the set of all rationals with odd denominator. This link is based on work by Allouche and Cosnard (see [4, 6, 7]), who related the set $\mathscr{U}(1)$ to kneading sequences of unimodal maps. The authors of [9] also explored a relationship between these sets and the real slice of the boundary of the Mandelbrot set.

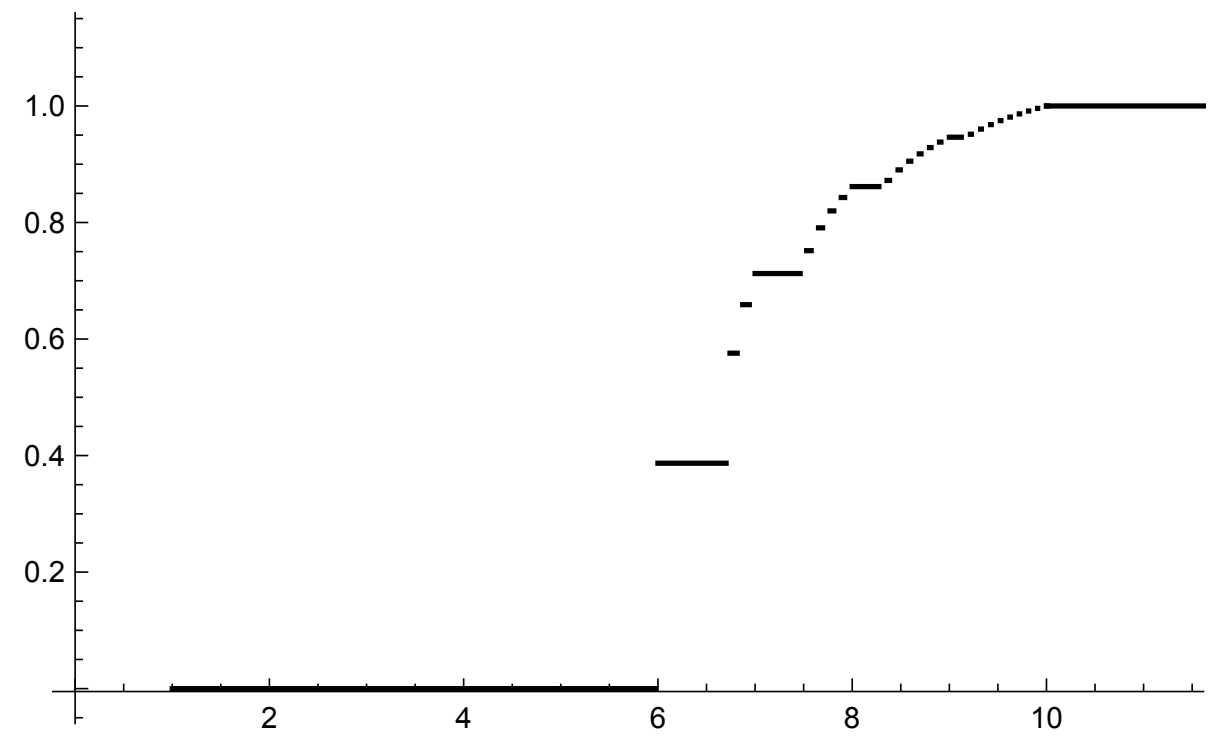

Figure 1. The asymptotic graph of the function $\phi(t)=\operatorname{dim}_{H}(\mathscr{U} \cap(1, t])$ for $t \in[4,11.5]$ with $M=9$ and $q_{K L}=q_{K L}(9) \approx 5.97592$.

By using Theorem 2 we investigate the dimensional spectrum of $\mathscr{U}$. Our next result strengthens the relationship between $\mathcal{U}_{q}$ and $\mathscr{U}$.

Theorem 3. For any $t>1$ we have

$$
\operatorname{dim}_{H}(\mathscr{U} \cap(1, t])=\max _{q \leq t} \operatorname{dim}_{H} \mathcal{U}_{q} .
$$

Moreover, the function $\phi(t):=\operatorname{dim}_{H}(\mathscr{U} \cap(1, t])$ is a Devil's staircase on $(1, \infty)$.

\section{Remark 1.3.}

(1) In 25] it was shown that $\overline{\mathscr{U}} \backslash \mathscr{U}$ is a countable set. Hence, Theorem 3 still holds if we replace $\mathscr{U}$ by $\overline{\mathscr{U}}$.

(2) Results from [23] (see Lemma 2.4 below) give that $\operatorname{dim}_{H} \mathcal{U}_{q}=1$ if and only if $q=$ $M+1$. In view of Theorem 3 we obtain that $\operatorname{dim}_{H}(\mathscr{U} \cap(1, t])<1$ for any $t<M+1$. 
This implies that the Hausdorff dimension of $\mathscr{U}$ is concentrated on the neighborhood of $M+1$.

As an application of Theorem 3 we investigate the variations of $\mathscr{U}=\mathscr{U}(M)$ when the parameter $M$ changes. For $K \in\{1,2, \ldots, M\}$, let $\mathscr{U}(K)$ be the set of bases $q \in(1, K+1]$ such that 1 has a unique $q$-expansion with respect to the alphabet $\{0,1, \ldots, K\}$. Theorem 4 characterizes the Hausdorff dimensions of the intersection $\mathscr{U}(M) \cap \mathscr{U}(K)$ and the difference $\mathscr{U}(M) \backslash \mathscr{U}(K)$. Indeed, we prove the following stronger result.

\section{Theorem 4 .}

(i) Let $K \in\{1,2, \ldots, M\}$. Then

$$
\operatorname{dim}_{H}\left(\bigcap_{J=K}^{M} \mathscr{U}(J)\right)=\max _{q \leq K+1} \operatorname{dim}_{H} \mathcal{U}_{q} .
$$

(ii) For any positive integer $L$ we have

$$
\operatorname{dim}_{H}\left(\mathscr{U}(L) \backslash \bigcup_{J \neq L} \mathscr{U}(J)\right)=1 .
$$

Remark 1.4. By the proof of Theorem 4 it follows that for $K<M$ the intersection

$$
\bigcap_{J=K}^{M} \mathscr{U}(J)=\mathscr{U}(M) \cap(1, K+1]
$$

is a proper subset of $\mathscr{U}(K)$. This, together with (1.3), implies that for $K<M$ neither the intersection $\bigcap_{J=K}^{M} \mathscr{U}(J)$ nor the difference set $\mathscr{U}(M) \backslash \bigcap_{J=K}^{M} \mathscr{U}(J)$ contains isolated points.

We emphasize that for each $q \in(1, M+1]$ the univoque set $\mathcal{U}_{q}$ is related to the dynamical system

$$
T_{q, j}:\left[0, \frac{M}{q-1}\right] \rightarrow\left[0, \frac{M}{q-1}\right] ; \quad x \mapsto q x-j
$$

for $j \in\{0,1, \ldots, M\}$. On the other hand, the set $\mathscr{U}$ contains all parameters $q \in(1, M+1]$ such that 1 has a unique $q$-expansion, and thus $\mathscr{U}$ is related to infinitely many dynamical systems. A similar set up involving a bifurcation set for infinitely many dynamical systems is considered in [9] (see also [10]). They considered the bifurcation set of an entropy map for a family of maps $\left\{T_{\alpha}:[\alpha-1, \alpha] \rightarrow[\alpha-1, \alpha]\right\}_{\alpha \in[0,1]}$, called $\alpha$-continued fraction transformations [32], where for each $\alpha \in[0,1]$ the map $T_{\alpha}$ is defined by

$$
T_{\alpha}(x)=\left\{\begin{array}{cl}
\frac{1}{|x|}-\left\lfloor\frac{1}{|x|}+1-\alpha\right\rfloor & \text { if } x \neq 0 \\
0 & \text { if } x=0
\end{array}\right.
$$


Each map $T_{\alpha}$ has a unique invariant measure $\mu_{\alpha}$ that is absolutely continuous with respect to the Lebesgue measure. They showed that the map

$$
\psi: \alpha \mapsto h_{\mu_{\alpha}}\left(T_{\alpha}\right)
$$

assigning to each $\alpha$ the measure theoretic entropy $h_{\mu_{\alpha}}\left(T_{\alpha}\right)$, has countably many intervals on which it is monotonic. The complement of the union of these intervals in $[0,1]$, i.e., the bifurcation set of $\psi$ denoted by $F$, has Lebesgue measure 0 (see [29] and [10]) and Hausdorff dimension 1 (see [9]). Moreover, in [9] the authors identified a homeomorphism between the set $F$ and the set $\Lambda \backslash\{0\}$ from (1.4), giving also a relation to the set $\mathscr{U}(1)$. In [9], however, no information is given on the local structure of $F$. Recently, Dajani and the first author identified in [12] another set $E$ that is linked to the sets $\mathscr{U}(1), \Lambda$ and $F$. They investigated a family of symmetric doubling maps $S_{\gamma}:[-1,1] \rightarrow[-1,1]$, given by

$$
S_{\gamma}(x)=2 x-\gamma\lfloor 2 x\rfloor,
$$

where $\lfloor x\rfloor$ denotes the integer part of $x$, and showd that the set $E$ of parameters $\gamma \in[1,2]$ for which the map $S_{\gamma}$ does not have a piecewise smooth invariant density is homeomorphic to $\Lambda \backslash\{0\}$. Therefore, the results obtained in this paper about the set $\mathscr{U}(1)$ can be used to investigate the bifurcation sets $E, F$ and the set $\Lambda$.

The rest of the paper is arranged in the following way. In Section 2 we fix the notation and recall some properties of unique $q$-expansions. Moreover, we recall from [1] some important properties of the bifurcation set $\mathscr{B}$. In Section 3 we give the proof of Theorem 1 for the dimensional homogeneousness of $\mathcal{U}_{q}$. In Section 4 we prove an auxiliary proposition that will be used to prove Theorem 2 in Section [5. The proof of Theorems 3 and 4 will be given in Sections 6 and 7, respectively. We end the paper with some remarks.

\section{UNIQUE EXPANSIONS AND BIFURCATION SET}

In this section we recall some properties of unique $q$-expansions and of the bifurcation set $\mathscr{B}$ as well. First we need some terminology from symbolic dynamics (cf. [30]).

2.1. Symbolic dynamics. Given a positive integer $M$, let $\{0,1, \ldots, M\}^{*}$ denote the set of all finite strings of symbols from $\{0,1, \ldots, M\}$, called words, together with the empty word denoted by $\epsilon$. Let $\{0,1, \ldots, M\}^{\mathbb{N}}$ be the set of sequences $\left(d_{i}\right)=d_{1} d_{2} \ldots$ with each element $d_{i} \in\{0,1, \ldots, M\}$. Let $\sigma$ be the left shift on $\{0,1, \ldots, M\}^{\mathbb{N}}$ defined by $\sigma\left(\left(d_{i}\right)\right)=\left(d_{i+1}\right)$. Then $\left(\{0,1, \ldots, M\}^{\mathbb{N}}, \sigma\right)$ is a full shift. For a word $\mathbf{c}=c_{1} \ldots c_{n} \in\{0,1, \ldots, M\}^{*}$ we denote by $\mathbf{c}^{k}=\left(c_{1} \ldots c_{n}\right)^{k}$ the $k$-fold concatenation of $\mathbf{c}$ to itself and by $\mathbf{c}^{\infty}=\left(c_{1} \ldots c_{n}\right)^{\infty}$ the periodic sequence with period block $\mathbf{c}$. Moreover, for a word $\mathbf{c}=c_{1} \ldots c_{n}$ with $c_{n}<M$ we 
denote by $\mathbf{c}^{+}$the word

$$
\mathbf{c}^{+}=c_{1} \ldots c_{n-1}\left(c_{n}+1\right) \text {. }
$$

Similarly, for a word $\mathbf{c}=c_{1} \ldots c_{n}$ with $c_{n}>0$ we write $\mathbf{c}^{-}=c_{1} \ldots c_{n-1}\left(c_{n}-1\right)$. For a sequence $\left(d_{i}\right) \in\{0,1, \ldots, M\}^{\mathbb{N}}$ we denote its reflection by

$$
\overline{\left(d_{i}\right)}=\left(M-d_{1}\right)\left(M-d_{2}\right) \cdots
$$

Accordingly, for a word $\mathbf{c}=c_{1} \ldots c_{n}$ we denote its reflection by $\overline{\mathbf{c}}=\left(M-c_{1}\right) \cdots\left(M-c_{n}\right)$.

On words and sequences we consider the lexicographical ordering $\prec, \preccurlyeq, \succ$ or $\succcurlyeq$ which is defined as follows. For two sequences $\left(c_{i}\right),\left(d_{i}\right) \in\{0,1, \ldots, M\}^{\mathbb{N}}$ we say that $\left(c_{i}\right) \prec\left(d_{i}\right)$ if there exists $n \in \mathbb{N}$ such that $c_{1} \ldots c_{n-1}=d_{1} \ldots d_{n-1}$ and $c_{n}<d_{n}$. Moreover, we write $\left(c_{i}\right) \preccurlyeq\left(d_{i}\right)$ if $\left(c_{i}\right) \prec\left(d_{i}\right)$ or $\left(c_{i}\right)=\left(d_{i}\right)$. Similarly, we write $\left(c_{i}\right) \succ\left(d_{i}\right)$ if $\left(d_{i}\right) \prec\left(c_{i}\right)$, and $\left(c_{i}\right) \succcurlyeq\left(d_{i}\right)$ if $\left(d_{i}\right) \preccurlyeq\left(c_{i}\right)$. We extend this definition to words in the following way. For two words $\omega, \nu \in\{0,1, \ldots, M\}^{*}$ we write $\omega \prec \nu$ if $\omega 0^{\infty} \prec \nu 0^{\infty}$. Accordingly, for a sequence $\left(d_{i}\right) \in\{0,1, \ldots, M\}^{\mathbb{N}}$ and a word $\mathbf{c}=c_{1} \ldots c_{m}$ we say $\left(d_{i}\right) \prec \mathbf{c}$ if $\left(d_{i}\right) \prec \mathbf{c} 0^{\infty}$.

Let $\mathcal{F} \subseteq\{0,1, \ldots, M\}^{*}$ and let $X=X_{\mathcal{F}} \subseteq\{0,1, \ldots, M\}^{\mathbb{N}}$ be the set of those sequences that do not contain any word from $\mathcal{F}$. We call the pair $(X, \sigma)$ a subshift. If $\mathcal{F}$ can be chosen to be a finite set, then $(X, \sigma)$ is called a subshift of finite type. For $n \in \mathbb{N} \cup\{0\}$ we denote by $\mathcal{L}_{n}(X)$ the set of words of length $n$ occurring in sequences of $X$. In particular, for $n=0$ we set $\mathcal{L}_{0}(X)=\{\epsilon\}$. The languange of $(X, \sigma)$ is then defined by

$$
\mathcal{L}(X)=\bigcup_{n=0}^{\infty} \mathcal{L}_{n}(X) .
$$

So, $\mathcal{L}(X)$ is the set of all finite words occurring in sequences from $X$.

For a subshift $(X, \sigma)$ and a word $\omega \in \mathcal{L}(X)$ let $F_{X}(\omega)$ be the follower set of $\omega$ in $X$ defined by

$$
F_{X}(\omega):=\left\{\left(d_{i}\right) \in X: d_{1} \ldots d_{|\omega|}=\omega\right\}
$$

where $|\mathbf{c}|$ denotes the length of a word $\mathbf{c} \in\{0,1, \ldots, M\}^{*}$.

A subshift $(X, \sigma)$ is called topologically transitive (or simply transitive) if for any two words $\omega, \nu \in \mathcal{L}(X)$ there exists a word $\gamma$ such that $\omega \gamma \nu \in \mathcal{L}(X)$. In other words, in a transitive subshift $(X, \sigma)$ any two words can be "connected" in $\mathcal{L}(X)$.

The topological entropy $h_{\text {top }}(X)$ of a subshift $(X, \sigma)$ is a quantity that indicates its complexity. It is defined by

$$
h_{\text {top }}(X)=\lim _{n \rightarrow \infty} \frac{\log \# \mathcal{L}_{n}(X)}{n}=\inf _{n \geq 1} \frac{\log \# \mathcal{L}_{n}(X)}{n},
$$


where $\# A$ denotes the cardinality of a set $A$. Accordingly, we define the topological entropy of a follower set $F_{X}(\omega)$ by changing $X$ to $F_{X}(\omega)$ in (2.2) if the corresponding limit exists. Clearly, if $X$ is a transitive subshift, then $h_{\text {top }}\left(F_{X}(\omega)\right)=h_{\text {top }}(X)$ for any $\omega \in \mathcal{L}(X)$.

2.2. Unique expansions. In this subsection we recall some results about unique expansions. For more information on this topic we refer the reader to the survey papers [36, 22] or the book chapter [16]. For $q \in(1, M+1]$, let

$$
\alpha(q)=\alpha_{1}(q) \alpha_{2}(q) \ldots
$$

be the quasi-greedy q-expansion of 1 (cf. [13]), i.e., the lexicographically largest $q$-expansion of 1 not ending with a string of zeros. The following characterization of quasi-greedy expansions was given in [8, Theorem 2.2].

Lemma 2.1. The map $q \mapsto \alpha(q)$ is a strictly increasing bijection from $(1, M+1]$ onto the set of all sequences $\left(a_{i}\right) \in\{0,1, \ldots, M\}^{\mathbb{N}}$ not ending with $0^{\infty}$ and satisfying

$$
a_{n+1} a_{n+2} \ldots \preceq a_{1} a_{2} \ldots \quad \text { whenever } a_{n}<M .
$$

Recall from (1.1) the definition of the projection map $\pi_{q}$ for $q \in(1, M+1]$ mapping $\{0,1, \ldots, M\}^{\mathbb{N}}$ onto the interval $I_{q, M}=[0, M /(q-1)]$. In general, $\pi_{q}$ is not bijective. However, $\pi_{q}$ is a bijection between $\mathbf{U}_{q}=\pi_{q}^{-1}\left(\mathcal{U}_{q}\right)$ and $\mathcal{U}_{q}$. The following lexicographical characterization of $\mathbf{U}_{q}$, or equivalently $\mathcal{U}_{q}$, was essentially due to Parry [33] (see also [8]).

Lemma 2.2. Let $q \in(1, M+1]$. Then $\left(x_{i}\right) \in \mathbf{U}_{q}$ if and only if

$$
\begin{aligned}
& x_{n+1} x_{n+2} \ldots \prec \alpha(q) \quad \text { whenever } x_{n}<M \text {, } \\
& \overline{x_{n+1} x_{n+2} \cdots} \prec \alpha(q) \quad \text { whenever } x_{n}>0 \text {. }
\end{aligned}
$$

Observe that $\mathscr{U}=\left\{q \in(1, M+1]: \alpha(q) \in \mathbf{U}_{q}\right\}$. As a corollary of Lemma 2.2 we have the following characterizations of $\mathscr{U}$ and $\overline{\mathscr{U}}$.

\section{Lemma 2.3.}

(i) $q \in \mathscr{U} \backslash\{M+1\}$ if and only if the quasi-greedy expansion $\alpha(q)$ satisfies

$$
\overline{\alpha(q)} \prec \sigma^{n}(\alpha(q)) \prec \alpha(q) \quad \text { for any } n \geq 1 .
$$

(ii) $q \in \overline{\mathscr{U}}$ if and only if the quasi-greedy expansion $\alpha(q)$ satisfies

$$
\overline{\alpha(q)} \prec \sigma^{n}(\alpha(q)) \preccurlyeq \alpha(q) \quad \text { for any } n \geq 1 \text {. }
$$

Proof. Part (i) was shown in [17, Theorem 2.5] and Part (ii) was proven in [17, Theorem $3.9]$. 
In [15] it was shown that $\left(\mathbf{U}_{q}, \sigma\right)$ is not necessarily a subshift. Inspired by 23] we consider the set $\mathbf{V}_{q}$ which contains all sequences $\left(x_{i}\right) \in\{0,1, \ldots, M\}^{\mathbb{N}}$ satisfying

$$
\overline{\alpha(q)} \preccurlyeq \sigma^{n}\left(\left(x_{i}\right)\right) \preccurlyeq \alpha(q) \quad \text { for all } \quad n \geq 0 \text {. }
$$

Then $\left(\mathbf{V}_{q}, \sigma\right)$ is a subshift (cf. [23, Lemma 2.6]). Furthermore, Lemma 2.1 implies that the set-valued map $q \mapsto \mathbf{V}_{q}$ is increasing, i.e., $\mathbf{V}_{p} \subseteq \mathbf{V}_{q}$ whenever $p<q$.

Recall that the Komornik-Loreti constant $q_{K L}$ is the smallest element of $\mathscr{U}$, which is defined in terms of the classical Thue-Morse sequence $\left(\tau_{i}\right)_{i=0}^{\infty}=01101001 \ldots$. Here the sequence $\left(\tau_{i}\right)_{i=0}^{\infty}$ is defined as follows (cf. [5]): $\tau_{0}=0$, and if $\tau_{0} \ldots \tau_{2^{n}-1}$ has already been defined for some $n \geq 0$, then $\tau_{2^{n}} \ldots \tau_{2^{n+1}-1}=\overline{\tau_{0} \ldots \tau_{2^{n}-1}}$. Then the Komornik-Loreti constant $q_{K L}=q_{K L}(M) \in(1, M+1]$ is the unique base satisfying

$$
\alpha\left(q_{K L}\right)=\lambda_{1} \lambda_{2} \ldots,
$$

where

$$
\lambda_{i}=\left\{\begin{array}{lll}
k+\tau_{i}-\tau_{i-1} & \text { if } \quad M=2 k, \\
k+\tau_{i} & \text { if } \quad M=2 k+1,
\end{array}\right.
$$

for each $i \geq 1$. We emphasize that the sequence $\left(\lambda_{i}\right)$ depends on $M$. By the definition of the Thue-Morse sequence $\left(\tau_{i}\right)_{i=0}^{\infty}$ it follows that (cf. [1])

$$
\lambda_{2^{n}+1} \ldots \lambda_{2^{n+1}}=\overline{\lambda_{1} \ldots \lambda_{2^{n}}}+\quad \text { for any } n \geq 0 .
$$

Recall that a function $f:[a, b] \rightarrow \mathbb{R}$ is called a Devil's staircase (or Cantor function) if $f$ is a continuous and non-decreasing function with $f(a)<f(b)$, and $f$ is locally constant almost everywhere. The next lemma summarizes some results from [23] on the Hausdorff dimension of $\mathcal{U}_{q}$.

\section{Lemma 2.4 .}

(i) For any $q \in(1, M+1]$ we have

$$
\operatorname{dim}_{H} \mathcal{U}_{q}=\frac{h_{t o p}\left(\mathbf{V}_{q}\right)}{\log q}
$$

(ii) The entropy function $H: q \mapsto h_{t o p}\left(\mathbf{V}_{q}\right)$ is a Devil's staircase in $(1, M+1]$ :

- $H$ is increasing and continuous in $(1, M+1]$;

- $H$ is locally constant almost everywhere in $(1, M+1]$;

- $H(q)=0$ if and only if $1<q \leq q_{K L}$. Moreover, $H(q)=\log (M+1)$ if and only if $q=M+1$.

Remark 2.5. 
(1) Lemma 2.4 implies that the dimensional function $D: q \mapsto \operatorname{dim}_{H} \mathcal{U}_{q}$ has a Devil's staircase behavior: (i) $D$ is continous in (1, M+1]; (ii) $D^{\prime}<0$ almost everywhere in $(1, M+1]$; (iii) $D(q)=0$ for any $q \in\left(1, q_{K L}\right]$ and $D(q)=1$ for $q=M+1$.

(2) In [23, Lemma 2.11] the authors showed that $H$ is locally constant on the complement of $\overline{\mathscr{U}}$, i.e., $H^{\prime}(q)=0$ for any $q \in(1, M+1] \backslash \overline{\mathscr{U}}$.

2.3. Bifurcation set. In this subsection we recall some recent results obtained in [1, where the authors investigated the maximal intervals on which $H$ is locally constant, called entropy plateaus (or simply called plateaus). For convenience of the reader we adopt much of the notation from [1]. We hope that this helps the interested reader who wants to access the relevant background information. Let $\mathscr{B}$ be the complement of these plateaus. From Lemma 2.4 (ii) we have

$$
\mathscr{B}=\{q \in(1, M+1]: H(p) \neq H(q) \text { for any } p \neq q\} .
$$

Note by $(1.2)$ that $\mathscr{B}$ is not closed. For the closure $\overline{\mathscr{B}}$ we have

$$
\overline{\mathscr{B}}=\{q \in(1, M+1]: \forall \delta>0, \exists p \in(q-\delta, q+\delta) \text { such that } H(p) \neq H(q)\} .
$$

In [1] $\overline{\mathscr{B}}$ was denoted by $\mathscr{E}$. The following lemma for $\overline{\mathscr{B}}$, the first part of which follows from Remark 2.5 (2), was established in [1, Theorem 3].

Lemma 2.6. $\overline{\mathscr{B}} \subset \overline{\mathscr{U}}$, and $\overline{\mathscr{B}}$ is a Cantor set of full Hausdorff dimension.

By Lemma 2.4 it follows that $\min \overline{\mathscr{B}}=q_{K L}$ and $\max \overline{\mathscr{B}}=M+1$. Since $\overline{\mathscr{B}}$ is a Cantor set, we can write

$$
\left(q_{K L}, M+1\right] \backslash \overline{\mathscr{B}}=\bigcup\left(p_{L}, p_{R}\right),
$$

where the union is pairwise disjoint and countable. By the definition of $\overline{\mathscr{B}}$ it follows that the intervals $\left[p_{L}, p_{R}\right]$ are the plateaus of $H$. In particular, since $H$ is increasing, these intervals have the property that $H(q)=H\left(p_{L}\right)$ if and only if $q \in\left[p_{L}, p_{R}\right]$. This implies that the bifurcation set $\mathscr{B}$ can be rewritten as in (1.2), i.e.,

$$
\mathscr{B}=\left(q_{K L}, M+1\right] \backslash \bigcup\left[p_{L}, p_{R}\right] .
$$

By (2.5) and (1.2) it follows that $\overline{\mathscr{B}} \backslash \mathscr{B}$ is countable. The fact that $\overline{\mathscr{B}}$ does not have isolated points gives the following lemma (see also [1]).

\section{Lemma 2.7.}

(i) For any $q \in\left(q_{K L}, M+1\right] \backslash \bigcup\left(p_{L}, p_{R}\right]$ there exists a sequence of plateaus $\left\{\left[p_{L}(n), p_{R}(n)\right]\right\}$ such that $p_{L}(n) \nearrow q$ as $n \rightarrow \infty$. 
(ii) For any $q \in\left[q_{K L}, M+1\right) \backslash \bigcup\left[p_{L}, p_{R}\right)$ there exists a sequence of plateaus $\left\{\left[q_{L}(n), q_{R}(n)\right]\right\}$ such that $q_{L}(n) \searrow q$ as $n \rightarrow \infty$.

So, by (2.5), (1.2) and Lemma 2.7 it follows that $\overline{\mathscr{B}} \backslash \mathscr{B}$ is a countable and dense subset of $\overline{\mathscr{B}}$. In particular, the set of left endpoints of all plateaus of $H$ is dense in $\overline{\mathscr{B}}$.

In [1] more detailed information on the structure of the plateaus of $H$ is given. Before we can give the necessary details, we have to recall some notation from [1]. Let $\mathbf{V}$ be the set of sequences $\left(a_{i}\right) \in\{0,1, \ldots, M\}^{\mathbb{N}}$ satisfying the inequalities

$$
\overline{\left(a_{i}\right)} \preccurlyeq \sigma^{n}\left(\left(a_{i}\right)\right) \preccurlyeq\left(a_{i}\right) \quad \text { for all } n \geq 0 .
$$

In [1, Lemma 3.3] it is proved that the subshift $\left(\mathbf{V}_{q}, \sigma\right)$ is not transitive for any $q \in\left(q_{K L}, q_{T}\right)$, where $q_{T} \in(1, M+1) \cap \mathscr{B}$ is the unique base such that

$$
\alpha\left(q_{T}\right)= \begin{cases}(k+1) k^{\infty} & \text { if } \quad M=2 k, \\ (k+1)((k+1) k)^{\infty} & \text { if } \quad M=2 k+1 .\end{cases}
$$

The plateaus of $H$ are characterized separately for the cases (A) $q \in\left[q_{T}, M+1\right]$ and (B) $q \in\left(q_{K L}, q_{T}\right)$.

(A). First we recall from [1] the following definition.

Definition 2.8. A sequence $\left(a_{i}\right) \in \mathbf{V}$ is called irreducible if

$$
a_{1} \ldots a_{j}\left({\overline{a_{1} \ldots a_{j}}}^{+}\right)^{\infty} \prec\left(a_{i}\right) \quad \text { whenever } \quad\left(a_{1} \ldots a_{j}^{-}\right)^{\infty} \in \mathbf{V} .
$$

Lemma 2.9. Let $\left[p_{L}, p_{R}\right] \subset\left[q_{T}, M+1\right]$ be a plateau of $H$.

(i) There exists a word $a_{1} \ldots a_{m} \in \mathcal{L}\left(\mathbf{V}_{p_{L}}\right)$ such that

$$
\alpha\left(p_{L}\right)=\left(a_{1} \ldots a_{m}\right)^{\infty} \text { is irreducible, and } \quad \alpha\left(p_{R}\right)=a_{1} \ldots a_{m}^{+}\left(\overline{a_{1} \ldots a_{m}}\right)^{\infty} .
$$

(ii) $\left(\mathbf{V}_{p_{L}}, \sigma\right)$ is a transitive subshift of finite type.

(iii) There exists a periodic sequence $\nu^{\infty} \in \mathbf{V}_{p_{L}}$ such that for any word $\eta \in \mathcal{L}\left(\mathbf{V}_{p_{L}}\right)$ we can find a large integer $N$ and a word $\omega$ satisfying

$$
\overline{\alpha_{1}\left(p_{L}\right) \ldots \alpha_{N}\left(p_{L}\right)} \prec \sigma^{j}\left(\eta \omega \nu^{\infty}\right) \prec \alpha_{1}\left(p_{L}\right) \ldots \alpha_{N}\left(p_{L}\right) \quad \text { for any } \quad j \geq 0 \text {. }
$$

Proof. Part (i) follows by [1, Proposition 5.2], and Part (ii) follows by [1, Lemma 5.1 (1)].

For (iii) we take

$$
\nu= \begin{cases}k & \text { if } \quad M=2 k, \\ (k+1) k & \text { if } \quad M=2 k+1 .\end{cases}
$$

Since $p_{L} \geq q_{T}$, by Lemma 2.1 we have $\alpha\left(p_{L}\right) \succcurlyeq \alpha\left(q_{T}\right)$. Then (2.6) gives that

$$
\overline{\alpha_{1}\left(p_{L}\right) \alpha_{2}\left(p_{L}\right)} \preccurlyeq \overline{\alpha_{1}\left(q_{T}\right) \alpha_{2}\left(q_{T}\right)} \prec \sigma^{j}\left(\nu^{\infty}\right) \prec \alpha_{1}\left(q_{T}\right) \alpha_{2}\left(q_{T}\right) \preccurlyeq \alpha_{1}\left(p_{L}\right) \alpha_{2}\left(p_{L}\right)
$$


for all $j \geq 0$. Note by (i) that $\alpha\left(p_{L}\right)$ is irreducible. By the proof of [1, Proposition 3.17] it follows that for any word $\eta \in \mathcal{L}\left(\mathbf{V}_{p_{L}}\right)$ there exist a large integer $N \geq 2$ and a word $\omega$ satisfying

$$
\overline{\alpha_{1}\left(p_{L}\right) \ldots \alpha_{N}\left(p_{L}\right)} \prec \sigma^{j}\left(\eta \omega \nu^{\infty}\right) \prec \alpha_{1}\left(p_{L}\right) \ldots \alpha_{N}\left(p_{L}\right) \quad \text { for any } \quad 0 \leq j<|\eta|+|\omega| .
$$

This together with (2.7) proves (iii).

(B). Now we consider plateaus of $H$ in $\left(q_{K L}, q_{T}\right)$. Let $\left(\lambda_{i}\right)$ be the quasi-greedy $q_{K L^{-}}$ expansion of 1 as given in (2.3). Note that $\left(\lambda_{i}\right)$ depends on $M$. For $n \geq 1$ let

$$
\xi(n)= \begin{cases}\lambda_{1} \ldots \lambda_{2^{n-1}}\left(\overline{\lambda_{1} \ldots \lambda_{2} 2^{n-1}}+\right)^{\infty} & \text { if } \quad M=2 k, \\ \lambda_{1} \ldots \lambda_{2^{n}}\left(\overline{\lambda_{1} \ldots \lambda_{2^{n}}}+\right)^{\infty} & \text { if } \quad M=2 k+1 .\end{cases}
$$

Then $\xi(1)=\alpha\left(q_{T}\right)$, and $\xi(n)$ is strictly decreasing to $\left(\lambda_{i}\right)=\alpha\left(q_{K L}\right)$ as $n \rightarrow \infty$. Moreover, [1, Lemma 4.2] gives that $\xi(n) \in \mathbf{V}$ for all $n \geq 1$. We recall from [1] the following definition.

Definition 2.10. A sequence $\left(a_{i}\right) \in \mathbf{V}$ is said to be $*$-irreducible if there exists $n \in \mathbb{N}$ such that $\xi(n+1) \preccurlyeq\left(a_{i}\right) \prec \xi(n)$, and

$$
a_{1} \ldots a_{j}\left(\overline{a_{1} \ldots a_{j}}+\right)^{\infty} \prec\left(a_{i}\right)
$$

whenever

$$
\left(a_{1} \ldots a_{j}^{-}\right)^{\infty} \in \mathbf{V} \quad \text { and } \quad j>\left\{\begin{array}{lll}
2^{n} & \text { if } \quad M=2 k, \\
2^{n+1} & \text { if } \quad M=2 k+1 .
\end{array}\right.
$$

Lemma 2.11. Let $\left[p_{L}, p_{R}\right] \subseteq\left(q_{K L}, q_{T}\right)$ be a plateau of $H$.

(i) There exists a word $a_{1} \ldots a_{m} \in \mathcal{L}\left(\mathbf{V}_{p_{L}}\right)$ such that

$$
\alpha\left(p_{L}\right)=\left(a_{1} \ldots a_{m}\right)^{\infty} \text { is } * \text {-irreducible, } \quad \text { and } \quad \alpha\left(p_{R}\right)=a_{1} \ldots a_{m}^{+}\left(\overline{a_{1} \ldots a_{m}}\right)^{\infty} .
$$

(ii) $\left(\mathbf{V}_{p_{L}}, \sigma\right)$ is a subshift of finite type, and it contains a unique transitive subshift of finite type $\left(X_{p_{L}}, \sigma\right)$ satisfying $h_{t o p}\left(X_{p_{L}}\right)=h_{t o p}\left(\mathbf{V}_{p_{L}}\right)$.

(iii) There exists a periodic sequence $\nu^{\infty} \in X_{p_{L}}$ such that for any word $\eta \in \mathcal{L}\left(\mathbf{V}_{p_{L}}\right)$ we can find a large integer $N$ and a word $\omega$ satisfying

$$
\overline{\alpha_{1}\left(p_{L}\right) \ldots \alpha_{N}\left(p_{L}\right)} \prec \sigma^{j}\left(\eta \omega \nu^{\infty}\right) \prec \alpha_{1}\left(p_{L}\right) \ldots \alpha_{N}\left(p_{L}\right) \quad \text { for any } j \geq 0 \text {. }
$$

Proof. Part (i) follows from [1, Proposition 5.11], and Part (ii) follows from [1, Lemma 5.9]. Then it remains to prove (iii).

By (i) we know that $\alpha\left(p_{L}\right)$ is a $*$-irreducible sequence. Then there exists $n \in \mathbb{N}$ such that $\xi(n+1) \preccurlyeq \alpha\left(p_{L}\right) \prec \xi(n)$. Note by (i) and (2.8) that $\alpha\left(p_{L}\right)$ is purely periodic while $\xi(n+1)$ is eventually periodic. Then $\alpha\left(p_{L}\right) \succ \xi(n+1)$. Let

$$
\nu= \begin{cases}\lambda_{1} \ldots \lambda_{2^{n}}^{-} & \text {if } \quad M=2 k \\ \lambda_{1} \ldots \lambda_{2^{n+1}}^{-} & \text {if } \quad M=2 k+1\end{cases}
$$


Then by the proof of [1, Lemma 5.9] we have $\nu^{\infty} \in X_{p_{L}}$. Observe by (2.4) and (2.8) that $\xi(n+1)=\nu^{+}(\bar{\nu})^{\infty} \in \mathbf{V}$. Then by using $\alpha\left(p_{L}\right) \succ \xi(n+1)$ it follows that there exists a large integer $N$ such that

$$
\overline{\alpha_{1}\left(p_{L}\right) \ldots \alpha_{N}\left(p_{L}\right)} \prec \sigma^{j}\left(\nu^{\infty}\right) \prec \alpha_{1}\left(p_{L}\right) \ldots \alpha_{N}\left(p_{L}\right) \quad \text { for any } \quad j \geq 0 \text {. }
$$

The remaining part of (iii) follows by the proof of [1, Lemma 5.8].

Finally, the following characterization of $\overline{\mathscr{B}}$ was established in [1, Theorem 3].

Lemma 2.12. $\overline{\mathscr{B}}=\overline{\left\{q \in\left(q_{K L}, M+1\right]: \alpha(q) \text { is irreducible or } * \text {-irreducible }\right\}}$.

\section{Dimensional homogeneity of $\mathcal{U}_{q}$}

In this section we will prove Theorem 1. Instead of proving Theorem 11 we prove the following equivalent statement.

Theorem 3.1. Let $q \in\left(1, q_{K L}\right] \cup\left(\left(q_{K L}, M+1\right] \backslash \bigcup\left(p_{L}, p_{R}\right]\right)$. Then for any $x \in \mathcal{U}_{q}$ we have

$$
\operatorname{dim}_{H}\left(\mathcal{U}_{q} \cap(x-\delta, x+\delta)\right)=\operatorname{dim}_{H} \mathcal{U}_{q} \quad \text { for any } \delta>0
$$

Before giving the proof of Theorem 3.1 we first explain why Theorem 3.1 is equivalent to Theorem 1. Clearly, Theorem 1 implies Theorem 3.1. On the other hand, take $q \in \mathscr{B}$. Let $V \subseteq \mathbb{R}$ be an open set with $\mathcal{U}_{q} \cap V \neq \emptyset$. Then there exist $x \in \mathcal{U}_{q} \cap V$ and $\delta>0$ such that

$$
\mathcal{U}_{q} \cap V \supset \mathcal{U}_{q} \cap(x-\delta, x+\delta) .
$$

By Theorem 3.1 it follows that $\operatorname{dim}_{H}\left(\mathcal{U}_{q} \cap V\right) \geq \operatorname{dim}_{H} \mathcal{U}_{q}$, which gives Theorem 1 ,

Note that for $q \in\left(1, q_{K L}\right]$ the statement of Theorem 3.1 follows immediately from the fact that $\operatorname{dim}_{H} \mathcal{U}_{q}=0$. For $q \in\left(q_{K L}, M+1\right]$ recall that $\mathbf{V}_{q}$ is the set of sequences $\left(x_{i}\right) \in$ $\{0,1, \ldots, M\}^{\mathbb{N}}$ satisfying

$$
\overline{\alpha(q)} \preccurlyeq \sigma^{n}\left(\left(x_{i}\right)\right) \preccurlyeq \alpha(q) \text { for all } n \geq 0 \text {. }
$$

Accordingly, let

$$
\mathcal{V}_{q}:=\left\{\pi_{q}\left(\left(x_{i}\right)\right):\left(x_{i}\right) \in \mathbf{V}_{q}\right\},
$$

where $\pi_{q}$ is the projection map defined in (1.1). For a set $A \subset \mathbb{R}$ and $r \in \mathbb{R}$ we denote by $r A:=\{r \cdot a: a \in A\}$ and $r+A:=\{r+a: a \in A\}$.

The following lemma for a relationship between $\mathcal{U}_{q}$ and $\mathcal{V}_{q}$ follows from Lemma 2.2 and the definition of $\mathcal{V}_{q}$. 
Lemma 3.2. Let $q \in\left(q_{K L}, M+1\right]$. Then $\mathcal{U}_{q}$ is a countable union of affine copies of $\mathcal{V}_{q}$ up to a countable set, i.e.,

$$
\begin{gathered}
\mathcal{U}_{q} \cup \mathcal{N}=\left\{0, \frac{M}{q-1}\right\} \cup \bigcup_{c_{1}=1}^{M-1}\left(\frac{c_{1}}{q}+\frac{\mathcal{V}_{q}}{q}\right) \cup \bigcup_{m=2}^{\infty} \bigcup_{c_{m}=1}^{M}\left(\frac{c_{m}}{q^{m}}+\frac{\mathcal{V}_{q}}{q^{m}}\right) \\
\cup \bigcup_{m=2}^{\infty} \bigcup_{c_{m}=0}^{M-1}\left(\sum_{i=1}^{m-1} \frac{M}{q^{i}}+\frac{c_{m}}{q^{m}}+\frac{\mathcal{V}_{q}}{q^{m}}\right),
\end{gathered}
$$

where the set $\mathcal{N}$ is at most countable.

Proof. For $q \in\left(q_{K L}, M+1\right]$ let $\mathbf{W}_{q}$ be the set of sequences $\left(x_{i}\right)$ satisfying

$$
\overline{\alpha(q)} \prec \sigma^{n}\left(\left(x_{i}\right)\right) \prec \alpha(q) \text { for any } n \geq 0,
$$

and let $\mathcal{W}_{q}=\pi_{q}\left(\mathbf{W}_{q}\right)$. Then $\mathcal{V}_{q} \backslash \mathcal{W}_{q}$ is at most countable (cf. [15]). By [23, Lemma 2.5] it follows that

$$
\begin{aligned}
\mathcal{U}_{q}=\{ & \left.0, \frac{M}{q-1}\right\} \cup \bigcup_{c_{1}=1}^{M-1}\left(\frac{c_{1}}{q}+\frac{\mathcal{W}_{q}}{q}\right) \cup \bigcup_{m=2}^{\infty} \bigcup_{c_{m}=1}^{M}\left(\frac{c_{m}}{q^{m}}+\frac{\mathcal{W}_{q}}{q^{m}}\right) \\
& \cup \bigcup_{m=2}^{\infty} \bigcup_{c_{m}=0}^{M-1}\left(\sum_{i=1}^{m-1} \frac{M}{q^{i}}+\frac{c_{m}}{q^{m}}+\frac{\mathcal{W}_{q}}{q^{m}}\right) .
\end{aligned}
$$

This establishes the lemma since $\mathcal{W}_{q} \subseteq \mathcal{V}_{q}$ and $\mathcal{V}_{q} \backslash \mathcal{W}_{q}$ is at most countable.

It immediately follows from Lemma 3.2 that

$$
\operatorname{dim}_{H} \mathcal{U}_{q}=\operatorname{dim}_{H} \mathcal{V}_{q} \quad \text { for any } q \in\left(q_{K L}, M+1\right]
$$

Hence, it suffices to prove Theorem 3.1 for $\mathcal{V}_{q}$ instead of $\mathcal{U}_{q}$. We first prove it for $q$ being the left endpoint of an entropy plateau.

Lemma 3.3. Let $\left[p_{L}, p_{R}\right] \subset\left(q_{K L}, M+1\right)$ be a plateau of $H$. Then for any $x \in \mathcal{V}_{p_{L}}$ we have

$$
\operatorname{dim}_{H}\left(\mathcal{V}_{p_{L}} \cap(x-\delta, x+\delta)\right)=\operatorname{dim}_{H} \mathcal{V}_{p_{L}} \quad \text { for any } \delta>0
$$

Proof. Obviously, $\operatorname{dim}_{H}\left(\mathcal{V}_{p_{L}} \cap(x-\delta, x+\delta)\right) \leq \operatorname{dim}_{H} \mathcal{V}_{p_{L}}$. So, it suffices to the prove the reverse inequality.

Fix $\delta>0$ and $x \in \mathcal{V}_{p_{L}}$. Suppose that $\left(x_{i}\right) \in \mathbf{V}_{p_{L}}$ is a $p_{L}$-expansion of $x$. Then there exists a large integer $N$ such that

$$
\pi_{p_{L}}\left(F_{\mathbf{V}_{p_{L}}}\left(x_{1} \ldots x_{N}\right)\right) \subseteq \mathcal{V}_{p_{L}} \cap(x-\delta, x+\delta),
$$

where the follower set $F_{\mathbf{V}_{p_{L}}}\left(x_{1} \ldots x_{N}\right)=\left\{\left(y_{i}\right) \in \mathbf{V}_{p_{L}}: y_{1} \ldots y_{N}=x_{1} \ldots x_{N}\right\}$ is as defined in (2.1). We split the proof into the following two cases. 
Case I. $\left[p_{L}, p_{R}\right] \subset\left[q_{T}, M+1\right]$. Then by Lemma 2.9 (ii) it follows that $\left(\mathbf{V}_{p_{L}}, \sigma\right)$ is a transitive subshift of finite type. This implies that

$$
h_{\text {top }}\left(F_{\mathbf{V}_{p_{L}}}\left(x_{1} \ldots x_{N}\right)\right)=h_{\text {top }}\left(\mathbf{V}_{p_{L}}\right) .
$$

Then, by (3.1), Lemma 2.4 (i) and Lemma 3.2 it follows that

$$
\begin{aligned}
\operatorname{dim}_{H}\left(\mathcal{V}_{p_{L}} \cap(x-\delta, x+\delta)\right) & \geq \operatorname{dim}_{H} \pi_{p_{L}}\left(F_{\mathbf{V}_{p_{L}}}\left(x_{1} \ldots x_{N}\right)\right) \\
& =\frac{h_{t o p}\left(F_{\mathbf{V}_{p_{L}}}\left(x_{1} \ldots x_{N}\right)\right)}{\log p_{L}} \\
& =\frac{h_{t o p}\left(\mathbf{V}_{p_{L}}\right)}{\log p_{L}}=\operatorname{dim}_{H} \mathcal{U}_{p_{L}}=\operatorname{dim}_{H} \mathcal{V}_{p_{L}} .
\end{aligned}
$$

Case II. $\left[p_{L}, p_{R}\right] \subset\left(q_{K L}, q_{T}\right)$. Then by Lemma2.11(ii) it follows that $\left(\mathbf{V}_{p_{L}}, \sigma\right)$ is a subshift of finite type that contains a unique transitive subshift of finite type $X_{p_{L}}$ such that

$$
h_{\text {top }}\left(X_{p_{L}}\right)=h_{\text {top }}\left(\mathbf{V}_{p_{L}}\right) .
$$

Furthermore, by Lemma 2.11 (iii) there exist a sequence $\nu^{\infty} \in X_{p_{L}}$ and a word $\omega$ such that

$$
x_{1} \ldots x_{N} \omega \nu^{\infty} \in F_{\mathbf{V}_{p_{L}}}\left(x_{1} \ldots x_{N}\right) .
$$

From [30, Proposition 2.1.7] there exists $m \geq 0$ such that $\left(\mathbf{V}_{p_{L}}, \sigma\right)$ is an $m$-step subshift of finite type. Note by (3.3) that the word $x_{1} \ldots x_{N} \omega \nu^{m} \in \mathcal{L}\left(\mathbf{V}_{p_{L}}\right)$. Then by [30, Theorem 2.1.8] it follows that for any sequence $\left(d_{i}\right) \in F_{X_{p_{L}}}\left(\nu^{m}\right) \subseteq F_{\mathbf{V}_{p_{L}}}\left(\nu^{m}\right)$ we have $x_{1} \ldots x_{N} \omega d_{1} d_{2} \ldots \in$ $F_{\mathbf{V}_{p_{L}}}\left(x_{1} \ldots x_{N}\right)$. In other words,

$$
\left\{x_{1} \ldots x_{N} \omega d_{1} d_{2} \ldots:\left(d_{i}\right) \in F_{X_{p_{L}}}\left(\nu^{m}\right)\right\} \subseteq F_{\mathbf{V}_{p_{L}}}\left(x_{1} \ldots x_{N}\right) .
$$

Therefore, by (3.1) it follows that

$$
\begin{aligned}
\operatorname{dim}_{H}\left(\mathcal{V}_{p_{L}} \cap(x-\delta, x+\delta)\right) & \geq \operatorname{dim}_{H} \pi_{p_{L}}\left(F_{\mathbf{V}_{p_{L}}}\left(x_{1} \ldots x_{N}\right)\right) \\
& \geq \operatorname{dim}_{H} \pi_{p_{L}}\left(F_{X_{p_{L}}}\left(\nu^{m}\right)\right)=\operatorname{dim}_{H} \pi_{p_{L}}\left(X_{p_{L}}\right),
\end{aligned}
$$

where the last equality holds by the transitivity of $\left(X_{p_{L}}, \sigma\right)$. Observe that $\pi_{p_{L}}\left(X_{p_{L}}\right)$ is a graph-directed set satisfying the open set condition (cf. [31]). Then the Hausdorff dimension of $\pi_{p_{L}}\left(X_{p_{L}}\right)$ is given by

$$
\operatorname{dim}_{H} \pi_{p_{L}}\left(X_{p_{L}}\right)=\frac{h_{t o p}\left(X_{p_{L}}\right)}{\log p_{L}} .
$$

By (3.2), (3.4), (3.5) and Lemma 2.4 (i) we conclude that

$$
\begin{aligned}
\operatorname{dim}_{H}\left(\mathcal{V}_{p_{L}} \cap(x-\delta, x+\delta)\right) & \geq \operatorname{dim}_{H} \pi_{p_{L}}\left(X_{p_{L}}\right) \\
& =\frac{h_{\text {top }}\left(X_{p_{L}}\right)}{\log p_{L}}=\frac{h_{\text {top }}\left(\mathbf{V}_{p_{L}}\right)}{\log p_{L}} \\
& =\operatorname{dim}_{H} \mathcal{U}_{p_{L}}=\operatorname{dim}_{H} \mathcal{V}_{p_{L}}
\end{aligned}
$$


Now we consider $q \in \mathscr{B}$. We need the following lemma.

Lemma 3.4. Let $q \in\left(q_{K L}, M+1\right]$ and $x_{1} \ldots x_{N} \in \mathcal{L}\left(\mathbf{V}_{q}\right)$. Let $\left\{p_{n}\right\} \subset(1, M+1]$ be a sequence such that $\alpha\left(p_{n}\right) \in \mathbf{V}$ for each $n \geq 1$, and $p_{n} \nearrow q$ as $n \rightarrow \infty$. Then

$$
x_{1} \ldots x_{N} \in \mathcal{L}\left(\mathbf{V}_{p_{n}}\right) \quad \text { for all sufficiently large } n \text {. }
$$

Proof. Since $x_{1} \ldots x_{N} \in \mathcal{L}\left(\mathbf{V}_{q}\right)$, we have

$$
\overline{\alpha_{1}(q) \ldots \alpha_{N-i}(q)} \preccurlyeq x_{i+1} \ldots x_{N} \preccurlyeq \alpha_{1}(q) \ldots \alpha_{N-i}(q) \quad \text { for any } \quad 0 \leq i<N .
$$

Let $s \in\{0,1, \ldots, N-1\}$ be the smallest integer such that

$$
x_{s+1} \ldots x_{N}=\overline{\alpha_{1}(q) \ldots \alpha_{N-s}(q)} \quad \text { or } \quad x_{s+1} \ldots x_{N}=\alpha_{1}(q) \ldots \alpha_{N-s}(q) .
$$

If there is no $s \in\{0,1, \ldots, N-1\}$ for which (3.6) holds, then we set $s=N$. By our choice of $s$ it follows that

$$
\overline{\alpha_{1}(q) \ldots \alpha_{N-i}(q)} \prec x_{i+1} \ldots x_{N} \prec \alpha_{1}(q) \ldots \alpha_{N-i}(q) \quad \text { for all } \quad 0 \leq i<s .
$$

In terms of (3.6) we may assume by symmetry that

$$
x_{s+1} \ldots x_{N}=\alpha_{1}(q) \ldots \alpha_{N-s}(q) .
$$

Since $p_{n} \nearrow q$ as $n \rightarrow \infty$, by Lemma 2.1 there exists $K \in \mathbb{N}$ such that

$$
\alpha_{1}\left(p_{n}\right) \ldots \alpha_{N}\left(p_{n}\right)=\alpha_{1}(q) \ldots \alpha_{N}(q) \quad \text { for any } n \geq K \text {. }
$$

By the assumption that $\alpha\left(p_{n}\right) \in \mathbf{V}$ for any $n \geq 1$, it follows from (3.7) and (3.8) that

$$
x_{1} \ldots x_{N} \alpha_{N-s+1}\left(p_{n}\right) \alpha_{N-s+2}\left(p_{n}\right) \ldots=x_{1} \ldots x_{s} \alpha_{1}\left(p_{n}\right) \alpha_{2}\left(p_{n}\right) \ldots \in \mathbf{V}_{p_{n}}
$$

for any $n \geq K$. So, $x_{1} \ldots x_{N} \in \mathcal{L}\left(\mathbf{V}_{p_{n}}\right)$ for all $n \geq K$.

Lemma 3.5. Let $q \in \mathscr{B}$. Then for any $x \in \mathcal{V}_{q}$ we have

$$
\operatorname{dim}_{H}\left(\mathcal{V}_{q} \cap(x-\delta, x+\delta)\right)=\operatorname{dim}_{H} \mathcal{V}_{q} \quad \text { for any } \delta>0
$$

Proof. Take $q \in \mathscr{B}$. Since $\mathscr{B} \subset\left(q_{K L}, M+1\right] \backslash \bigcup\left(p_{L}, p_{R}\right]$, by Lemma 2.7 (i) there exists a sequence of plateaus $\left\{\left[p_{L}(n), p_{R}(n)\right]\right\}_{n=1}^{\infty}$ such that $p_{L}(n) \nearrow q$ as $n \rightarrow \infty$.

Now we fix $\delta>0$ and $x \in \mathcal{V}_{q}$. Suppose $\left(x_{i}\right) \in \mathbf{V}_{q}$ is a $q$-expansion of $x$. Then there exists a large integer $N$ such that

$$
\pi_{q}\left(F_{\mathbf{V}_{q}}\left(x_{1} \ldots x_{N}\right)\right) \subseteq \mathcal{V}_{q} \cap(x-\delta, x+\delta) .
$$

By Lemmas 2.9 (i) and 2.11 (i) we have $\alpha\left(p_{L}(n)\right) \in \mathbf{V}$ for all $n \geq 1$. Then applying Lemma 3.4 to the sequence $\left\{p_{L}(n)\right\}$ gives a large integer $K$ such that

$$
x_{1} \ldots x_{N} \in \mathcal{L}\left(\mathbf{V}_{p_{L}(n)}\right) \quad \text { for all } n \geq K .
$$


Since $\mathbf{V}_{p_{L}(n)} \subset \mathbf{V}_{q}$ for any $n \geq 1$, it follows from (3.9) that

$$
\pi_{q}\left(F_{\mathbf{V}_{p_{L}(n)}}\left(x_{1} \ldots x_{N}\right)\right) \subset \mathcal{V}_{q} \cap(x-\delta, x+\delta) \quad \text { for all } n \geq K .
$$

By (3.10) and the proof of Lemma 3.3 it follows that for any $n \geq K$,

$$
\operatorname{dim}_{H}\left(\mathcal{V}_{q} \cap(x-\delta, x+\delta)\right) \geq \operatorname{dim}_{H} \pi_{q}\left(F_{\mathbf{V}_{p_{L}(n)}}\left(x_{1} \ldots x_{N}\right)\right) \geq \frac{h_{t o p}\left(\mathbf{V}_{p_{L}(n)}\right)}{\log q}
$$

Letting $n \rightarrow \infty$ we have $p_{L}(n) \nearrow q$, and then we conclude by the continuity of the function $q \mapsto h_{\text {top }}\left(\mathbf{V}_{q}\right)$ (see Lemma 2.4 (ii)) that

$$
\operatorname{dim}_{H}\left(\mathcal{V}_{q} \cap(x-\delta, x+\delta)\right) \geq \frac{h_{\text {top }}\left(\mathbf{V}_{q}\right)}{\log q}=\operatorname{dim}_{H} \mathcal{U}_{q}=\operatorname{dim}_{H} \mathcal{V}_{q}
$$

Proof of Theorem [3.1. Take $q \in\left(1, q_{K L}\right] \cup\left(\left(q_{K L}, M+1\right] \backslash \bigcup\left(p_{L}, p_{R}\right]\right)$. If $q \in\left(1, q_{K L}\right]$, then the result follows from the fact that $\operatorname{dim}_{H} \mathcal{U}_{q}=0$ (see Lemma 2.4).

Assume $q \in\left(q_{K L}, M+1\right] \backslash \bigcup\left(p_{L}, p_{R}\right]$ where the union is taken over all plateaus $\left[p_{L}, p_{R}\right]$ of $H$. Take $x \in \mathcal{U}_{q}$. If $x \notin\{0, M /(q-1)\}$, then by Lemma $3.2 x$ belongs to an affine copy of $\mathcal{V}_{q}$. Since the Hausdorff dimension is invariant under affine transformations (cf. [20]), the statement follows from Lemmas 3.3 and 3.5 .

So, it remains to consider $x=0$ and $x=M /(q-1)$. By symmetry we may assume $x=0$. Take $\delta>0$. Then by Lemma 3.2 there exists a sufficiently large integer $m$ such that

$$
\frac{1}{q^{m}}+\frac{\mathcal{V}_{q}}{q^{m}} \subseteq\left(\mathcal{U}_{q} \cup \mathcal{N}\right) \cap(-\delta, \delta),
$$

where $\mathcal{N}$ is at most countable. This proves the statement for $x=0$.

At the end of this section we strengthen Theorem 3.1 and give a complete characterization of the set

$$
\left\{q \in(1, M+1]: \mathcal{U}_{q} \text { is dimensionally homogeneous }\right\} .
$$

Let $\left[p_{L}, p_{R}\right] \subset\left(q_{K L}, M+1\right]$ be a plateau of $H$. Note that $p_{L} \in \overline{\mathscr{B}} \backslash \mathscr{B} \subset \overline{\mathscr{U}} \backslash \mathscr{U}$. Then by [15. Theorem 1.7] there exists a largest $\hat{p}_{L} \in\left(p_{L}, p_{R}\right)$ such that the set-valued map $q \mapsto \mathbf{V}_{q}$ is constant in $\left[p_{L}, \hat{p}_{L}\right)$. Furthermore, for $q=\hat{p}_{L}$ any sequence in the difference set $\mathbf{V}_{\hat{p}_{L}} \backslash \mathbf{V}_{p_{L}}$ is not contained in $\mathbf{U}_{\hat{p}_{L}}$. Then by the same argument as in the proof of Lemma 3.3 it follows that Theorem 3.1 also holds for any $q \in\left[p_{L}, \hat{p}_{L}\right]$. Clearly, $\mathcal{U}_{q}$ is dimensionally homogeneous for $q \leq q_{K L}$. So, the univoque set $\mathcal{U}_{q}$ is dimensionally homogeneous for any $q \in\left(1, q_{K L}\right] \cup$ $\left(\left(q_{K L}, M+1\right] \backslash \bigcup\left(\hat{p}_{L}, p_{R}\right]\right)$. This, combined with some recent progress obtained by Allaart et al. [2], implies the following.

\section{Theorem 3.6.}

(i) If $M=1$ or $M$ is even, then $\mathcal{U}_{q}$ is dimensionally homogeneous if, and only if, $q \in$ $\left(1, q_{K L}\right] \cup\left(\left(q_{K L}, M+1\right] \backslash \bigcup\left(\hat{p}_{L}, p_{R}\right]\right)$. 
(ii) If $M=2 k+1 \geq 3$, then $\mathcal{U}_{q}$ is dimensionally homogeneous if, and only if, $q \in\left(1, q_{K L}\right] \cup$ $\left(\left(q_{K L}, M+1\right] \backslash \bigcup\left(\hat{p}_{L}, p_{R}\right]\right)$ or $q=\frac{k+3+\sqrt{k^{2}+6 k+1}}{2}$.

Proof. By Theorem 3.1 and the above arguments it follows that $\mathcal{U}_{q}$ is dimensionally homogeneous for any $q \in\left(1, q_{K L}\right] \cup\left(\left(q_{K L}, M+1\right] \backslash \bigcup\left(\hat{p}_{L}, p_{R}\right]\right)$. Then to prove the sufficiency it remains to prove the dimensional homogeneity of $\mathcal{U}_{q}$ for $q=\frac{k+3+\sqrt{k^{2}+6 k+1}}{2}=: q_{\star}$ with $M=2 k+1 \geq 3$. Note that $q_{\star}$ is the right endpoint of the entropy plateau generated by $k+1$, i.e., $\left[p_{\star}, q_{\star}\right]$ is an entropy plateau with $\alpha\left(p_{\star}\right)=(k+1)^{\infty}$ and $\alpha\left(q_{\star}\right)=(k+2) k^{\infty}$. Then by [2, Corollary 3.10] it follows that

$$
h_{t o p}\left(\mathbf{V}_{q_{\star}} \backslash \mathbf{V}_{p_{\star}}\right)=h_{t o p}\left(\mathbf{V}_{p_{\star}}\right)=\log 2,
$$

where the second equality follows from that $\mathbf{V}_{p_{\star}}=\{k, k+1\}^{\mathbb{N}}$. Furthermore, any sequence in $\mathbf{V}_{q_{\star}} \backslash \mathbf{V}_{p_{\star}}$ eventually ends in a transitive sub-shift of finite type $(X, \sigma)$ with states $\{k-1, k, k+1, k+2\}$ and adjacency matrix

$$
A=\left(\begin{array}{llll}
0 & 0 & 1 & 1 \\
1 & 1 & 0 & 0 \\
0 & 0 & 1 & 1 \\
1 & 1 & 0 & 0
\end{array}\right) .
$$

Observe that $h_{t o p}(X)=\log 2$. Using (3.11) and by a similar argument as in the proof of Lemma 3.3 it follows that $\mathcal{U}_{q_{\star}}$ is dimensionally homogeneous.

Now we prove the necessity. Without loss of generality we assume that $M=1$ or $M$ is even. Let $\left[p_{L}, p_{R}\right] \subset\left(q_{K L}, M+1\right]$ be an entropy plateau generated by $a_{1} \ldots a_{m}$, and let $\hat{p}_{L} \in\left(p_{L}, p_{R}\right)$ be the largest point such that the map $q \mapsto \mathbf{V}_{q}$ is constant in $\left[p_{L}, \hat{p}_{L}\right)$. In fact, we have $\alpha\left(\hat{p}_{L}\right)=\left(a_{1} \ldots a_{m}^{+} \overline{a_{1} \ldots a_{m}^{+}}\right)^{\infty}$ (cf. [15]). Take $q \in\left(\hat{p}_{L}, p_{R}\right]$. Then $\mathbf{W}_{q} \backslash \mathbf{V}_{p_{L}} \neq \emptyset$, where $\mathbf{W}_{q}$ is the set of sequences $\left(x_{i}\right)$ satisfying

$$
\overline{\alpha(q)} \prec \sigma^{n}\left(\left(x_{i}\right)\right) \prec \alpha(q) \text { for any } n \geq 0 \text {. }
$$

Furthermore, any sequence in $\mathbf{W}_{q} \backslash \mathbf{V}_{p_{L}}$ must end in the sub-shift of finite type $(Y, \sigma)$ with states $\left\{\overline{a_{1} \ldots a_{m}^{+}}, \overline{a_{1} \ldots a_{m}}, a_{1} \ldots a_{m}, a_{1} \ldots a_{m}^{+}\right\}$and adjacency matrix $A$ defined in (3.12). In particular,

$$
h_{\text {top }}(Y)=\frac{\log 2}{m}=h_{\text {top }}\left(\mathbf{V}_{p_{R}} \backslash \mathbf{V}_{p_{L}}\right)<h_{\text {top }}\left(\mathbf{V}_{p_{L}}\right),
$$

where the inequality follows from [2, Corollary 3.10]. Observe that $\mathbf{W}_{q} \subseteq \mathbf{U}_{q}$. Therefore, by (3.13) and the same argument as in the proof of Lemma 3.3 it follows that for any $x \in$ $\pi_{q}\left(\mathbf{W}_{q} \backslash \mathbf{V}_{p_{L}}\right) \subset \mathcal{U}_{q}$ there exists $\delta>0$ such that

$$
\operatorname{dim}_{H}\left(\mathcal{U}_{q} \cap(x-\delta, x+\delta)\right) \leq \frac{h_{t o p}(Y)}{\log q}<\frac{h_{t o p}\left(\mathbf{V}_{p_{L}}\right)}{\log q}=\operatorname{dim}_{H} \mathcal{U}_{q}
$$


This completes the proof.

\section{Auxiliary Proposition}

In this section we prove an auxiliary proposition that will be used to prove Theorem 2 in the next section.

Proposition 4.1. Let $q \in \overline{\mathscr{B}} \backslash\{M+1\}$. Then for any $\varepsilon>0$ there exists $\delta>0$ such that

$$
(1-\varepsilon) \operatorname{dim}_{H} \pi_{q}\left(\mathbf{B}_{\delta}(q)\right) \leq \operatorname{dim}_{H}(\overline{\mathscr{B}} \cap(q-\delta, q+\delta)) \leq(1+\varepsilon) \operatorname{dim}_{H} \pi_{q+\delta}\left(\mathbf{B}_{\delta}(q)\right),
$$

where

$$
\mathbf{B}_{\delta}(q):=\{\alpha(p): p \in \overline{\mathscr{B}} \cap(q-\delta, q+\delta)\}
$$

The proof of Proposition 4.1 is based on the following lemma for the Hausdorff dimension under Hölder continuous maps (cf. [20]).

Lemma 4.2. Let $f:\left(X, \rho_{1}\right) \rightarrow\left(Y, \rho_{2}\right)$ be a Hölder map between two metric spaces, i.e., there exist two constants $C>0$ and $\lambda>0$ such that

$$
\rho_{2}(f(x), f(y)) \leq C \rho_{1}(x, y)^{\lambda}
$$

for any $x, y \in X$ with $\rho_{1}(x, y) \leq c$ (here $c$ is a small constant). Then $\operatorname{dim}_{H} f(X) \leq \frac{1}{\lambda} \operatorname{dim}_{H} X$.

First we prove the second inequality in Proposition 4.1.

Lemma 4.3. Let $q \in \overline{\mathscr{B}} \backslash\{M+1\}$. Then for any $\varepsilon>0$ there exists $\delta>0$ such that

$$
\operatorname{dim}_{H}(\overline{\mathscr{B}} \cap(q-\delta, q+\delta)) \leq(1+\varepsilon) \operatorname{dim}_{H} \pi_{q+\delta}\left(\mathbf{B}_{\delta}(q)\right) .
$$

Proof. Fix $\varepsilon>0$ and $q \in \overline{\mathscr{B}} \backslash\{M+1\}$. Then there exists $\delta>0$ such that

$$
q-\delta>1, \quad q+\delta<M+1 \quad \text { and } \quad \frac{\log (q+\delta)}{\log (q-\delta)} \leq 1+\varepsilon .
$$

Since $\overline{\mathscr{B}} \subseteq \overline{\mathscr{U}}$, by Lemmas 2.1 and 2.3 (ii) it follows that for each $p \in \overline{\mathscr{B}} \cap(q-\delta, q+\delta$ ) we have

$$
\overline{\alpha(q+\delta)} \prec \overline{\alpha(p)} \prec \sigma^{i}(\alpha(p)) \preccurlyeq \alpha(p) \prec \alpha(q+\delta) \quad \text { for all } \quad i \geq 0 .
$$

So, by Lemma $2.2 \alpha(p) \in \mathbf{U}_{q+\delta}$ for any $p \in \overline{\mathscr{B}} \cap(q-\delta, q+\delta)$. This implies that the map

$$
g: \overline{\mathscr{B}} \cap(q-\delta, q+\delta) \rightarrow \pi_{q+\delta}\left(\mathbf{B}_{\delta}(q)\right) ; \quad p \mapsto \pi_{q+\delta}(\alpha(p))
$$

is bijective. By Lemma 4.2 it suffices to prove that there exists a constant $C>0$ such that

$$
\left|\pi_{q+\delta}\left(\alpha\left(p_{2}\right)\right)-\pi_{q+\delta}\left(\alpha\left(p_{1}\right)\right)\right| \geq C\left|p_{2}-p_{1}\right|^{1+\varepsilon}
$$

for any $p_{1}, p_{2} \in \overline{\mathscr{B}} \cap(q-\delta, q+\delta)$. 
Take $p_{1}, p_{2} \in \overline{\mathscr{B}} \cap(q-\delta, q+\delta)$ with $p_{1}<p_{2}$. Then by Lemma 2.1 we have $\alpha\left(p_{1}\right) \prec \alpha\left(p_{2}\right)$. So, there exists $n \geq 1$ such that

$$
\alpha_{1}\left(p_{1}\right) \ldots \alpha_{n-1}\left(p_{1}\right)=\alpha_{1}\left(p_{2}\right) \ldots \alpha_{n-1}\left(p_{2}\right) \text { and } \alpha_{n}\left(p_{1}\right)<\alpha_{n}\left(p_{2}\right) .
$$

Then

$$
\begin{aligned}
0<p_{2}-p_{1} & =\sum_{i=1}^{\infty} \frac{\alpha_{i}\left(p_{2}\right)}{p_{2}^{i-1}}-\sum_{i=1}^{\infty} \frac{\alpha_{i}\left(p_{1}\right)}{p_{1}^{i-1}} \\
& \leq \sum_{i=1}^{n-1}\left(\frac{\alpha_{i}\left(p_{2}\right)}{p_{2}^{i-1}}-\frac{\alpha_{i}\left(p_{1}\right)}{p_{1}^{i-1}}\right)+\sum_{i=n}^{\infty} \frac{\alpha_{i}\left(p_{2}\right)}{p_{2}^{i-1}} \leq p_{2}^{2-n},
\end{aligned}
$$

where the last inequality follows from the property of quasi-greedy expansion $\alpha\left(p_{2}\right)$ that $\sum_{i=1}^{\infty} \alpha_{k+i}\left(p_{2}\right) / p_{2}^{i} \leq 1$ for any $k \geq 1$.

On the other hand, by (4.1) we have $\alpha\left(p_{2}\right) \preccurlyeq \alpha(q+\delta) \prec \alpha(M+1)=M^{\infty}$. Then there exists a large integer $N$ (depending on $q+\delta$ ) such that

$$
\alpha_{1}\left(p_{2}\right) \ldots \alpha_{N}\left(p_{2}\right) \preccurlyeq M^{N-1}(M-1) .
$$

Note that $p_{2} \in \overline{\mathscr{B}} \subseteq \overline{\mathscr{U}}$. Then by Lemma 2.3 (ii) and (4.3) it follows that

$$
\alpha_{m+1}\left(p_{2}\right) \alpha_{m+2}\left(p_{2}\right) \ldots \succ \overline{\alpha\left(p_{2}\right)} \succcurlyeq 0^{N-1} 10^{\infty} \quad \text { for any } \quad m \geq 1 .
$$

This implies that

$$
\begin{aligned}
& \pi_{q+\delta}\left(\alpha\left(p_{2}\right)\right)-\pi_{q+\delta}\left(\alpha\left(p_{1}\right)\right) \\
= & \sum_{i=1}^{\infty} \frac{\alpha_{i}\left(p_{2}\right)-\alpha_{i}\left(p_{1}\right)}{(q+\delta)^{i}} \\
= & \frac{\alpha_{n}\left(p_{2}\right)-\alpha_{n}\left(p_{1}\right)}{(q+\delta)^{n}}-\frac{1}{(q+\delta)^{n}} \sum_{i=1}^{\infty} \frac{\alpha_{n+i}\left(p_{1}\right)}{(q+\delta)^{i}}+\sum_{i=n+1}^{\infty} \frac{\alpha_{i}\left(p_{2}\right)}{(q+\delta)^{i}} \\
\geq & \frac{1}{(q+\delta)^{n}}-\frac{1}{(q+\delta)^{n}} \sum_{i=1}^{\infty} \frac{\alpha_{n+i}\left(p_{1}\right)}{p_{1}^{i}}+\sum_{i=n+1}^{\infty} \frac{\alpha_{i}\left(p_{2}\right)}{(q+\delta)^{i}} \\
\geq & \sum_{i=n+1}^{\infty} \frac{\alpha_{i}\left(p_{2}\right)}{(q+\delta)^{i}} \geq \frac{1}{(q+\delta)^{n+N}},
\end{aligned}
$$

where the second inequality follows from the same property of the quasi-greedy expansion $\alpha\left(p_{1}\right)$ that was used before.

Therefore, by (4.1) and (4.2) it follows that

$$
\begin{aligned}
\pi_{q+\delta}\left(\alpha\left(p_{2}\right)\right)-\pi_{q+\delta}\left(\alpha\left(p_{1}\right)\right) & \geq\left((q+\delta)^{-\frac{n+N}{1+\varepsilon}}\right)^{1+\varepsilon} \\
& \geq\left((q-\delta)^{-n-N}\right)^{1+\varepsilon} \\
& \geq(q-\delta)^{-N(1+\varepsilon)}\left(p_{2}^{-n}\right)^{1+\varepsilon} \geq C\left(p_{2}-p_{1}\right)^{1+\varepsilon}
\end{aligned}
$$


where the constant $C=(q-\delta)^{-N(1+\varepsilon)}(q+\delta)^{-2(1+\varepsilon)}$. This completes the proof.

Now we turn to prove the first inequality of Proposition 4.1.

Lemma 4.4. Let $q \in \overline{\mathscr{B}} \backslash\{M+1\}$. Then for any $\varepsilon>0$ there exists $\delta>0$ such that

$$
\operatorname{dim}_{H}(\overline{\mathscr{B}} \cap(q-\delta, q+\delta)) \geq(1-\varepsilon) \operatorname{dim}_{H} \pi_{q}\left(\mathbf{B}_{\delta}(q)\right) .
$$

Proof. The proof is similar to that of Lemma 4.3. Fix $\varepsilon>0$ and take $q \in \overline{\mathscr{B}} \backslash\{M+1\}$. Then there exists $\delta>0$ such that

$$
q-\delta>1, \quad q+\delta<M+1 \quad \text { and } \quad \frac{\log (q+\delta)}{\log q} \leq \frac{1}{1-\varepsilon} .
$$

Take $p_{1}, p_{2} \in \overline{\mathscr{B}} \cap(q-\delta, q+\delta)$ with $p_{1}<p_{2}$. Then by Lemma 2.1 we have $\alpha\left(p_{1}\right) \prec \alpha\left(p_{2}\right)$, and therefore there exists a smallest integer $n \geq 1$ such that $\alpha_{n}\left(p_{1}\right)<\alpha_{n}\left(p_{2}\right)$. This implies that

$$
\left|\pi_{q}\left(\alpha\left(p_{2}\right)\right)-\pi_{q}\left(\alpha\left(p_{1}\right)\right)\right|=\left|\sum_{i=1}^{\infty} \frac{\alpha_{i}\left(p_{2}\right)-\alpha_{i}\left(p_{1}\right)}{q^{i}}\right| \leq \sum_{i=n}^{\infty} \frac{M}{q^{i}}=\frac{M q}{q-1} q^{-n} .
$$

On the other hand, observe that $q+\delta<M+1$. Then $\alpha\left(p_{2}\right) \preccurlyeq \alpha(q+\delta) \prec \alpha(M+1)=M^{\infty}$. So, there exists $N \geq 1$ such that

$$
\alpha_{1}\left(p_{2}\right) \ldots \alpha_{N}\left(p_{2}\right) \preccurlyeq M^{N-1}(M-1) .
$$

Since $p_{2} \in \overline{\mathscr{B}} \subseteq \overline{\mathscr{U}}$, Lemma 2.3 (ii) gives

$$
1=\sum_{i=1}^{\infty} \frac{\alpha_{i}\left(p_{2}\right)}{p_{2}^{i}}>\sum_{i=1}^{n} \frac{\alpha_{i}\left(p_{2}\right)}{p_{2}^{i}}+\frac{1}{p_{2}^{n+N}}
$$

which implies that

$$
\begin{aligned}
\frac{1}{p_{2}^{n+N}} & <1-\sum_{i=1}^{n} \frac{\alpha_{i}\left(p_{2}\right)}{p_{2}^{i}}=\sum_{i=1}^{\infty} \frac{\alpha_{i}\left(p_{1}\right)}{p_{1}^{i}}-\sum_{i=1}^{n} \frac{\alpha_{i}\left(p_{2}\right)}{p_{2}^{i}} \\
& \leq \sum_{i=1}^{n}\left(\frac{\alpha_{i}\left(p_{2}\right)}{p_{1}^{i}}-\frac{\alpha_{i}\left(p_{2}\right)}{p_{2}^{i}}\right) \\
& \leq \sum_{i=1}^{\infty}\left(\frac{M}{p_{1}^{i}}-\frac{M}{p_{2}^{i}}\right)=\frac{M}{\left(p_{1}-1\right)\left(p_{2}-1\right)}\left(p_{2}-p_{1}\right) .
\end{aligned}
$$

Here the second inequality holds since

$$
\begin{aligned}
& \alpha_{1}\left(p_{1}\right) \ldots \alpha_{n-1}\left(p_{1}\right)=\alpha_{1}\left(p_{2}\right) \ldots \alpha_{n-1}\left(p_{2}\right) \\
& \alpha_{n}\left(p_{1}\right)<\alpha_{n}\left(p_{2}\right) \text { and } \sum_{i=1}^{\infty} \alpha_{n+i}\left(p_{1}\right) / p_{1}^{i} \leq 1
\end{aligned}
$$


Therefore, by (4.4)-(4.6) we conclude that

$$
\begin{aligned}
\left|\pi_{q}\left(\alpha\left(p_{2}\right)\right)-\pi_{q}\left(\alpha\left(p_{1}\right)\right)\right| & \leq \frac{M q^{N+1}}{q-1}\left(q^{-\frac{n+N}{1-\varepsilon}}\right)^{1-\varepsilon} \\
& \leq \frac{M q^{N+1}}{q-1}(q+\delta)^{-(n+N)(1-\varepsilon)} \\
& \leq \frac{M q^{N+1}}{q-1} p_{2}^{-(n+N)(1-\varepsilon)}<C\left(p_{2}-p_{1}\right)^{1-\varepsilon}
\end{aligned}
$$

where

$$
C=\frac{M^{2-\varepsilon} q^{N+1}}{(q-1)(q-\delta-1)^{2(1-\varepsilon)}} .
$$

Note by Lemma 2.1 that the map $p \mapsto \alpha(p)$ is bijective from $\overline{\mathscr{B}} \cap(q-\delta, q+\delta)$ onto $\mathbf{B}_{\delta}(q)$. Hence, the lemma follows by letting $f=\pi_{q} \circ \alpha$ in Lemma 4.2 ,

Proof of Proposition 4.1. The proposition follows from Lemmas 4.3 and 4.4 .

\section{LOCAL DIMENSION OF $\mathscr{B}$}

In this section we will prove Theorem 2, which states that for any $q \in \overline{\mathscr{B}}$ we have

$$
\lim _{\delta \rightarrow 0} \operatorname{dim}_{H}(\overline{\mathscr{B}} \cap(q-\delta, q+\delta))=\operatorname{dim}_{H} \mathcal{U}_{q}
$$

First we prove the upper bound.

Proposition 5.1. For any $q \in \overline{\mathscr{B}}$ we have

$$
\lim _{\delta \rightarrow 0} \operatorname{dim}_{H}(\overline{\mathscr{B}} \cap(q-\delta, q+\delta)) \leq \operatorname{dim}_{H} \mathcal{U}_{q}
$$

Proof. Take $q \in \overline{\mathscr{B}}$. By Lemma 2.4 and Proposition 4.1 it follows that for any $\varepsilon>0$ there exists a $\delta>0$ such that

$$
\begin{aligned}
\operatorname{dim}_{H} \mathcal{U}_{q+\delta} & \leq \operatorname{dim}_{H} \mathcal{U}_{q}+\varepsilon, \\
\operatorname{dim}_{H}(\overline{\mathscr{B}} \cap(q-\delta, q+\delta)) & \leq(1+\varepsilon) \operatorname{dim}_{H} \pi_{q+\delta}\left(\mathbf{B}_{\delta}(q)\right),
\end{aligned}
$$

where $\mathbf{B}_{\delta}(q)=\{\alpha(p): p \in(q-\delta, q+\delta) \cap \overline{\mathscr{B}}\}$.

Since $\overline{\mathscr{B}} \subseteq \overline{\mathscr{U}}$, Lemmas 2.1 and 2.3 (ii) give that any sequence $\alpha(p) \in \mathbf{B}_{\delta}(q)$ satisfies

$$
\overline{\alpha(q+\delta)} \prec \overline{\alpha(p)} \prec \sigma^{n}(\alpha(p)) \preccurlyeq \alpha(p) \prec \alpha(q+\delta) \quad \text { for all } n \geq 0 \text {. }
$$

By Lemma 2.2 this implies that $\mathbf{B}_{\delta}(q) \subseteq \mathbf{U}_{q+\delta}$. Therefore, by (5.1) it follows that

$$
\begin{aligned}
\operatorname{dim}_{H}(\overline{\mathscr{B}} \cap(q-\delta, q+\delta)) & \leq(1+\varepsilon) \operatorname{dim}_{H} \pi_{q+\delta}\left(\mathbf{B}_{\delta}(q)\right) \\
& \leq(1+\varepsilon) \operatorname{dim}_{H} \mathcal{U}_{q+\delta} \leq(1+\varepsilon)\left(\operatorname{dim}_{H} \mathcal{U}_{q}+\varepsilon\right)
\end{aligned}
$$

Since $\varepsilon>0$ was arbitrary, this completes the proof. 
The proof of the lower bound of Theorem 2 is tedious. We will prove this in several steps. First we need the following lemma.

Lemma 5.2. Let $\left[p_{L}, p_{R}\right] \subseteq\left(q_{K L}, M+1\right)$ be a plateau of $H$ such that $\alpha\left(p_{L}\right)=\left(\alpha_{1} \ldots \alpha_{m}\right)^{\infty}$ with period $m$. Then

$$
\begin{aligned}
& \alpha_{i+1} \ldots \alpha_{m} \prec \alpha_{1} \ldots \alpha_{m-i} \quad \text { for all } 0<i<m \text {, } \\
& \alpha_{i+1} \ldots \alpha_{m} \alpha_{1} \ldots \alpha_{i} \succ \overline{\alpha_{1} \ldots \alpha_{m}} \quad \text { for all } \quad 0 \leq i<m \text {. }
\end{aligned}
$$

Proof. Since $\left(\alpha_{1} \ldots \alpha_{m}\right)^{\infty}$ is the quasi-greedy $p_{L^{-}}$expansion of 1 with period $m$, the greedy $p_{L^{-}}$ expansion of 1 is $\alpha_{1} \ldots \alpha_{m}^{+} 0^{\infty}$. So, by [17, Propostion 2.2] it follows that $\sigma^{n}\left(\alpha_{1} \ldots \alpha_{m}^{+} 0^{\infty}\right) \prec$ $\alpha_{1} \ldots \alpha_{m}^{+} 0^{\infty}$ for any $n \geq 1$. This implies

$$
\alpha_{i+1} \ldots \alpha_{m} \prec \alpha_{i+1} \ldots \alpha_{m}^{+} \preccurlyeq \alpha_{1} \ldots \alpha_{m-i} \quad \text { for any } \quad 0<i<m .
$$

Lemma 2.6 states that $p_{L} \in \overline{\mathscr{B}} \subset \overline{\mathscr{U}}$. Then by Lemma 2.3 (ii) we have that

$$
\left(\alpha_{i+1} \ldots \alpha_{m} \alpha_{1} \ldots \alpha_{i}\right)^{\infty}=\sigma^{i}\left(\left(\alpha_{1} \ldots \alpha_{m}\right)^{\infty}\right) \succ\left(\overline{\alpha_{1} \ldots \alpha_{m}}\right)^{\infty}
$$

for any $0 \leq i<m$. This implies that

$$
\alpha_{i+1} \ldots \alpha_{m} \alpha_{1} \ldots \alpha_{i} \succ \overline{\alpha_{1} \ldots \alpha_{m}} \quad \text { for any } 0 \leq i<m .
$$

Let $\left[p_{L}, p_{R}\right] \subset\left(q_{K L}, M+1\right)$ be a plateau of $H$. For any $N \geq 1$ let $\left(\mathbf{W}_{p_{L}, N}, \sigma\right)$ be a subshift of finite type in $\{0,1, \ldots, M\}^{\mathbb{N}}$ with the set of forbidden blocks $c_{1} \ldots c_{N}$ satisfying

$$
c_{1} \ldots c_{N} \preccurlyeq \overline{\alpha_{1}\left(p_{L}\right) \ldots \alpha_{N}\left(p_{L}\right)} \quad \text { or } \quad c_{1} \ldots c_{N} \succcurlyeq \alpha_{1}\left(p_{L}\right) \ldots \alpha_{N}\left(p_{L}\right) .
$$

Then any sequence $\left(x_{i}\right) \in \mathbf{W}_{p_{L}, N}$ satisfies

$$
\overline{\alpha_{1}\left(p_{L}\right) \ldots \alpha_{N}\left(p_{L}\right)} \prec \sigma^{n}\left(\left(x_{i}\right)\right) \prec \alpha_{1}\left(p_{L}\right) \ldots \alpha_{N}\left(p_{L}\right) \quad \text { for all } \quad n \geq 0 .
$$

If $\alpha_{N}\left(p_{L}\right)>0$, then $\mathbf{W}_{p_{L}, N}$ is indeed the set of sequences $\left(x_{i}\right) \in\{0,1, \ldots, M\}^{\mathbb{N}}$ satisfying

$$
\left(\overline{\alpha_{1}\left(p_{L}\right) \ldots \alpha_{N}\left(p_{L}\right)}\right)^{+} \preccurlyeq \sigma^{n}\left(\left(x_{i}\right)\right) \preccurlyeq\left(\alpha_{1}\left(p_{L}\right) \ldots \alpha_{N}\left(p_{L}\right)^{-}\right)^{\infty}
$$

for all $n \geq 0$. By the definition of $\mathbf{W}_{p_{L}, N}$ it gives that

$$
\mathbf{W}_{p_{L}, 1} \subseteq \mathbf{W}_{p_{L}, 2} \subseteq \cdots \subseteq \mathbf{V}_{p_{L}}
$$

We emphasize that $\mathbf{W}_{p_{L}, 1}$ can be an empty set, and the inclusions in the above equation are not necessarily strict.

Observe that $\left(\mathbf{V}_{p_{L}}, \sigma\right)$ is a subshift of finite type with positive topological entropy. The following asymptotic result was established in [23, Proposition 2.8].

Lemma 5.3. Let $\left[p_{L}, p_{R}\right] \subseteq\left[q_{T}, M+1\right]$ be a plateau of $H$. Then

$$
\lim _{N \rightarrow \infty} h_{\text {top }}\left(\mathbf{W}_{p_{L}, N}\right)=h_{\text {top }}\left(\mathbf{V}_{p_{L}}\right) \text {. }
$$


Recall from (2.8) that

$$
\begin{array}{lr}
\xi(n)=\lambda_{1} \ldots \lambda_{2^{n-1}}\left(\overline{\lambda_{1} \ldots \lambda_{2^{n-1}}}+\right)^{\infty} & \text { if } \quad M=2 k, \\
\xi(n)=\lambda_{1} \ldots \lambda_{2^{n}}\left(\overline{\lambda_{1} \ldots \lambda_{2^{n}}}+\right)^{\infty} & \text { if } \quad M=2 k+1 .
\end{array}
$$

Note that the sequence $\left(\lambda_{i}\right)$ in the definition of $\xi(n)$ depends on $M$. In the following lemma we show that the entropy of $\left(\mathbf{W}_{p_{L}, N}, \sigma\right)$ is equal to the entropy of the follower set $F_{\mathbf{W}_{p_{L}, N}}(\nu)$ for all sufficiently large integers $N$, where $\nu$ is the word defined in Lemma 2.9 (iii) or Lemma 2.11 (iii).

\section{Lemma 5.4.}

(i) Let $\left[p_{L}, p_{R}\right] \subset\left[q_{T}, M+1\right]$ be a plateau of $H$, and let

$$
\nu= \begin{cases}k & \text { if } \quad M=2 k, \\ (k+1) k & \text { if } \quad M=2 k+1 .\end{cases}
$$

Then for all sufficiently large integers $N$ we have

$$
h_{\text {top }}\left(F_{\mathbf{W}_{p_{L}, N}}\left(\nu^{\ell}\right)\right)=h_{\text {top }}\left(\mathbf{W}_{p_{L}, N}\right) \quad \text { for any } \quad \ell \geq 1 .
$$

(ii) Let $\left[p_{L}, p_{R}\right] \subset\left(q_{K L}, q_{T}\right)$ be a plateau of $H$ with $\xi(n+1) \preccurlyeq \alpha\left(p_{L}\right) \prec \xi(n)$. Set

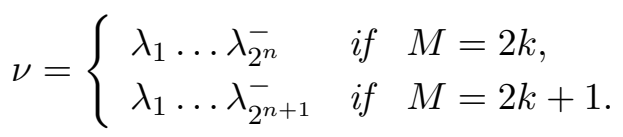

Then for all sufficiently large integers $N$ we have

$$
h_{\text {top }}\left(F_{\mathbf{W}_{p_{L}, N}}\left(\nu^{\ell}\right)\right)=h_{\text {top }}\left(\mathbf{W}_{p_{L}, N}\right) \quad \text { for any } \quad \ell \geq 1 .
$$

Proof. Take $\ell \geq 1$. First we prove (i). By Lemma 2.9 (iii) there exists a large integer $N \geq 2$ such that $\nu^{\ell} \in \mathcal{L}\left(\mathbf{W}_{p_{L}, N}\right)$. Since $\left(\mathbf{W}_{p_{L}, N}, \sigma\right)$ is a subshift of finite type, to prove (i) it suffices to prove that for any word $\rho \in \mathcal{L}\left(\mathbf{W}_{p_{L}, N}\right)$ there exists a word $\gamma$ of uniformly bounded length for which $\nu^{\ell} \gamma \rho \in \mathcal{L}\left(\mathbf{W}_{p_{L}, N}\right)$.

Take $\rho=\rho_{1} \ldots \rho_{m} \in \mathcal{L}\left(\mathbf{W}_{p_{L}, N}\right)$. If $M=2 k$, then $\nu=k$. Since $\alpha\left(p_{L}\right) \succcurlyeq \alpha\left(q_{T}\right)=(k+1) k^{\infty}$, we have

$$
\overline{\alpha_{1}\left(p_{L}\right)} \leq k-1<\nu<k+1 \leq \alpha_{1}\left(p_{L}\right) .
$$

So, $\nu^{\ell} \gamma \rho \in \mathcal{L}\left(\mathbf{W}_{p_{L}, N}\right)$ by taking $\gamma=\epsilon$ the empty word. Similarly, if $M=2 k+1$ then $\nu=(k+1) k$. Observe that $\alpha\left(p_{L}\right) \succcurlyeq \alpha\left(q_{T}\right)=(k+1)((k+1) k)^{\infty}$. This implies that $\nu^{\ell} \gamma \rho \in$ $\mathcal{L}\left(\mathbf{W}_{p_{L}, N}\right)$ by taking $\gamma=\epsilon$ if the initial word $\rho_{1} \geq k+1$, and by taking $\gamma=k+1$ if $\rho_{1} \leq k$.

Now we turn to prove (ii). We only give the proof for $M=2 k$, since the proof for $M=2 k+1$ is similar. Then $\nu=\lambda_{1} \ldots \lambda_{2^{n}}^{-}$. By Lemma 2.11 (iii) there exists a large integer $N \geq 2^{n+1}$ such that $\nu^{\infty}=\left(\lambda_{1} \ldots \lambda_{2^{n}}^{-}\right)^{\infty} \in \mathbf{W}_{p_{L}, N}$. Since $h_{t o p}\left(\mathbf{V}_{p_{L}}\right)>0$, by Lemma 5.3 we can choose $N$ sufficiently large such that $h_{\text {top }}\left(\mathbf{W}_{p_{L}, N}\right)>0$. Since $\mathbf{W}_{p_{L}, N}$ is a subshift of finite type, there 
exists a transitive subshift of finite type $X_{N} \subset \mathbf{W}_{p_{L}, N}$ for which $h_{\text {top }}\left(X_{N}\right)=h_{\text {top }}\left(\mathbf{W}_{p_{L}, N}\right)$ (cf. [30, Theorem 4.4.4]). We claim that the word $\lambda_{1} \ldots \lambda_{2^{n}}$ or $\overline{\lambda_{1} \ldots \lambda_{2^{n}}}$ belongs to $\mathcal{L}\left(X_{N}\right)$.

By (2.8) and (2.4) it follows that

$$
\xi(n)=\lambda_{1} \ldots \lambda_{2^{n-1}}\left(\overline{\lambda_{1} \ldots \lambda_{2^{n-1}}}+\right)^{\infty}=\lambda_{1} \ldots \lambda_{2^{n}}\left(\overline{\lambda_{1} \ldots \lambda_{2^{n-1}}}+\right)^{\infty} .
$$

Then the assumption $\xi(n+1) \preccurlyeq \alpha\left(p_{L}\right) \prec \xi(n)$ gives that

$$
\alpha_{1}\left(p_{L}\right) \ldots \alpha_{2^{n}}\left(p_{L}\right)=\lambda_{1} \ldots \lambda_{2^{n}}=\alpha_{1}\left(q_{K L}\right) \ldots \alpha_{2^{n}}\left(q_{K L}\right) .
$$

Suppose that the words $\lambda_{1} \ldots \lambda_{2^{n}}$ and $\overline{\lambda_{1} \ldots \lambda_{2^{n}}}$ do not belong to $\mathcal{L}\left(X_{N}\right)$. Then by (5.2) we have

$$
X_{N} \subset \mathbf{W}_{p_{L}, 2^{n}}=\mathbf{W}_{q_{K L}, 2^{n}} \subset \mathbf{V}_{q_{K L}} .
$$

So, by Lemma 2.4 it follows that $X_{N}$ has zero topological entropy, leading to a contradiction with $h_{\text {top }}\left(X_{N}\right)=h_{\text {top }}\left(\mathbf{W}_{p_{L}, N}\right)>0$.

By the claim, to finish the proof of (ii) it suffices to prove that for any word $\rho \in \mathcal{L}\left(X_{N}\right)$ with a prefix $\lambda_{1} \ldots \lambda_{2^{n}}$ or $\overline{\lambda_{1} \ldots \lambda_{2^{n}}}$ there exists a word $\gamma$ of uniformly bounded length such that $\nu^{\ell} \gamma \rho \in \mathcal{L}\left(\mathbf{W}_{p_{L}, N}\right)$. In [26, Lemma 4.2] (see also, [1, Lemma 4.2]) it was shown that for any $n \geq 1$ we have

$$
\overline{\lambda_{1} \ldots \lambda_{2^{n}-i}} \prec \lambda_{i+1} \ldots \lambda_{2^{n}} \preccurlyeq \lambda_{1} \ldots \lambda_{2^{n}-i} \quad \text { for any } \quad 0 \leq i<2^{n} .
$$

This implies that for any $0 \leq i<2^{n}$ we have

$$
\lambda_{i+1} \ldots \lambda_{2^{n}}^{-} \prec \lambda_{1} \ldots \lambda_{2^{n}-i} \quad \text { and } \quad \lambda_{i+1} \ldots \lambda_{2^{n}}^{-} \lambda_{1} \ldots \lambda_{i} \succ \overline{\lambda_{1} \ldots \lambda_{2^{n}}} .
$$

Observe that

$$
\nu=\lambda_{1} \ldots \lambda_{2^{n}}^{-}=\lambda_{1} \ldots \lambda_{2^{n-1}} \overline{\lambda_{1} \ldots \lambda_{2^{n-1}}} .
$$

Then by (5.2) and (5.3) it follows that if $\lambda_{1} \ldots \lambda_{2^{n}}$ is a prefix of $\rho$, then $\nu^{\ell} \gamma \rho \in \mathcal{L}\left(\mathbf{W}_{p_{L}, N}\right)$ by taking $\gamma=\epsilon$ the empty word, and if $\overline{\lambda_{1} \ldots \lambda_{2^{n}}}$ is a prefix of $\rho$ then $\nu^{\ell} \gamma \rho \in \mathcal{L}\left(\mathbf{W}_{p_{L}, N}\right)$ by taking $\gamma=\lambda_{1} \ldots \lambda_{2^{n-1}}$.

In the following lemma we prove the lower bound of Theorem 2 for $q \in\left[q_{T}, M+1\right]$ being the left endpoint of an entropy plateau.

Lemma 5.5. Let $\left[p_{L}, p_{R}\right] \subseteq\left[q_{T}, M+1\right]$ be a plateau of $H$. Then for any $\delta>0$ we have

$$
\operatorname{dim}_{H}\left(\overline{\mathscr{B}} \cap\left(p_{L}-\delta, p_{L}+\delta\right)\right) \geq \operatorname{dim}_{H} \mathcal{U}_{p_{L}}
$$

Proof. By Lemma 2.9 (i) it follows that $\alpha\left(p_{L}\right)=\left(\alpha_{i}\right)=\left(\alpha_{1} \ldots \alpha_{m}\right)^{\infty}$ is an irreducible sequence, where $m$ is the minimal period of $\alpha\left(p_{L}\right)$. Then, there exists a large integer $N_{1}>m$ such that

$$
\alpha_{1} \cdots \alpha_{j}\left({\overline{\alpha_{1} \ldots \alpha_{j}}}^{+}\right)^{\infty} \prec \alpha_{1} \ldots \alpha_{N_{1}} \quad \text { if } \quad\left(\alpha_{1} \ldots \alpha_{j}^{-}\right)^{\infty} \in \mathbf{V} \text { and } 1 \leq j \leq m .
$$


Let $\nu$ be the word defined in Lemma 5.4 (i). Then by Lemma 2.9 (iii) there exist a large integer $N>N_{1}$ and a word $\omega$ such that

$$
\overline{\alpha_{1} \ldots \alpha_{N}} \prec \sigma^{n}\left(\alpha_{1} \ldots \alpha_{m} \omega \nu^{\infty}\right) \prec \alpha_{1} \ldots \alpha_{N} \quad \text { for any } \quad n \geq 0 \text {. }
$$

Observe that $\left(\mathbf{W}_{p_{L}, N}, \sigma\right)$ is an $N$-step subshift of finite type. Note by (5.5) that $\alpha_{1} \ldots \alpha_{m} \omega \nu^{N} \in$ $\mathcal{L}\left(\mathbf{W}_{p_{L}, N}\right)$. Then by [30, Theorem 2.1.8] it follows that for any sequence $\left(d_{i}\right) \in F_{\mathbf{W}_{p_{L}, N}}\left(\nu^{N}\right)$ we have $\alpha_{1} \ldots \alpha_{m} \omega d_{1} d_{2} \ldots \in F_{\mathbf{W}_{p_{L}, N}}\left(\alpha_{1} \ldots \alpha_{m}\right)$. In other words,

$$
\left\{\alpha_{1} \ldots \alpha_{m} \omega d_{1} d_{2} \ldots:\left(d_{i}\right) \in F_{\mathbf{W}_{p_{L}, N}}\left(\nu^{N}\right)\right\} \subseteq F_{\mathbf{W}_{p_{L}, N}}\left(\alpha_{1} \ldots \alpha_{m}\right) \subseteq \mathbf{W}_{p_{L}, N}
$$

So,

$$
h_{\text {top }}\left(F_{\mathbf{W}_{p_{L}, N}}\left(\nu^{N}\right)\right) \leq h_{\text {top }}\left(F_{\mathbf{W}_{p_{L}, N}}\left(\alpha_{1} \ldots \alpha_{m}\right)\right) \leq h_{\text {top }}\left(\mathbf{W}_{p_{L}, N}\right) .
$$

Therefore, by Lemma 5.4 (i) we obtain

$$
h_{t o p}\left(F_{\mathbf{W}_{p_{L}, N}}\left(\alpha_{1} \ldots \alpha_{m}\right)\right)=h_{t o p}\left(\mathbf{W}_{p_{L}, N}\right) .
$$

Let $\Lambda_{N}$ be the set of sequences $\left(a_{i}\right) \in\{0,1, \ldots, M\}^{\infty}$ satisfying

$$
a_{1} \ldots a_{m N}=\left(\alpha_{1} \ldots \alpha_{m}\right)^{N} \quad \text { and } \quad a_{m N+1} a_{m N+2} \ldots \in F_{\mathbf{W}_{p_{L}, N}}\left(\alpha_{1} \ldots \alpha_{m}\right) .
$$

Fix $\delta>0$. We claim that

$$
\Lambda_{N} \subseteq \mathbf{B}_{\delta}\left(p_{L}\right)=\left\{\alpha(q): q \in \overline{\mathscr{B}} \cap\left(p_{L}-\delta, p_{L}+\delta\right)\right\}
$$

for all sufficiently large integers $N>N_{1}$.

Clearly, when $N$ increases the length of the common prefix of sequences in $\Lambda_{N}$ grows, and it coincides with a prefix of $\alpha\left(p_{L}\right)=\left(\alpha_{1} \ldots \alpha_{m}\right)^{\infty}$. So, by Lemmas 2.1 and 2.12 it suffices to show that for all $N>N_{1}$ any sequence $\left(a_{i}\right) \in \Lambda_{N}$ is irreducible.

Take $N>N_{1}$ and $\left(a_{i}\right) \in \Lambda_{N}$. First we claim that

$$
\overline{\alpha_{1} \ldots \alpha_{N}} \prec \sigma^{n}\left(\left(a_{i}\right)\right) \prec \alpha_{1} \ldots \alpha_{N} \quad \text { for any } n \geq 1 \text {. }
$$

Observe that $a_{1} \ldots a_{m N}=\left(\alpha_{1} \ldots \alpha_{m}\right)^{N}$ and the tails $a_{m N+1} a_{m N+2} \ldots \in F_{\mathbf{W}_{p_{L}, N}}\left(\alpha_{1} \ldots \alpha_{m}\right)$. Since $N>N_{1}>m$, (5.7) follows directly from Lemma 5.2.

Note by the definition of $\Lambda_{N}$ that $a_{1} \ldots a_{N}=\alpha_{1} \ldots \alpha_{N}$. By (5.7) it follows that $\left(a_{i}\right) \in \mathbf{V}$. So, by Definition 2.8 it remains to prove that

$$
a_{1} \ldots a_{j}\left({\overline{a_{1} \ldots a_{j}}}^{+}\right)^{\infty} \prec\left(a_{i}\right) \quad \text { whenever } \quad\left(a_{1} \ldots a_{j}^{-}\right)^{\infty} \in \mathbf{V} .
$$

We split the proof of (5.8) into the following three cases.

- For $1 \leq j \leq m$, (5.8) follows from (5.4). 
- For $m<j \leq N$, let $j=j_{1} m+r_{1}$ with $j_{1} \geq 1$ and $r_{1} \in\{1,2, \ldots, m\}$. Since $\left(\alpha_{1} \ldots \alpha_{j}^{-}\right)^{\infty}=\left(\left(\alpha_{1} \ldots \alpha_{m}\right)^{j_{1}} \alpha_{1} \ldots \alpha_{r_{1}}^{-}\right)^{\infty} \in \mathbf{V}$, we have

$$
\alpha_{r_{1}+1} \ldots \alpha_{m} \alpha_{1} \ldots \alpha_{r_{1}} \succ \alpha_{r_{1}+1} \ldots \alpha_{m} \alpha_{1} \ldots \alpha_{r_{1}}^{-} \succcurlyeq \overline{\alpha_{1} \ldots \alpha_{m}} .
$$

This implies that

$$
\begin{aligned}
a_{1} \ldots a_{j}\left(\overline{a_{1} \ldots a_{j}}\right)^{+\infty} & =\left(\alpha_{1} \ldots \alpha_{m}\right)^{j_{1}} \alpha_{1} \ldots \alpha_{r_{1}} \overline{\alpha_{1} \ldots \alpha_{m}} \ldots \\
& \prec\left(\alpha_{1} \ldots \alpha_{m}\right)^{j_{1}} \alpha_{1} \ldots \alpha_{r_{1}} \alpha_{r_{1}+1} \ldots \alpha_{m} \alpha_{1} \ldots \alpha_{r_{1}} 0^{\infty} \\
& \preccurlyeq\left(a_{i}\right) .
\end{aligned}
$$

- For $j>N$, by (5.7) it follows that

$$
\left({\overline{a_{1} \ldots a_{j}}}^{+}\right)^{\infty}=\left({\overline{\alpha_{1} \ldots \alpha_{N} a_{N+1} \ldots a_{j}}}^{+}\right)^{\infty} \prec a_{j+1} a_{j+2} \ldots,
$$

which implies that (5.8) also holds in this case.

Therefore, $\left(a_{i}\right)$ is an irreducible sequence, and thus $\left(a_{i}\right) \in \mathbf{B}_{\delta}\left(p_{L}\right)$. So, $\Lambda_{N} \subseteq \mathbf{B}_{\delta}\left(p_{L}\right)$ for all $N>N_{1}$.

Note that $\pi_{p_{L}}\left(\Lambda_{N}\right)$ is a scaling copy of $\pi_{p_{L}}\left(F_{\mathbf{W}_{p_{L}, N}}\left(\alpha_{1} \ldots \alpha_{m}\right)\right)$ which is related to a graphdirected set satisfying the open set condition (cf. [23, Lemma 3.2]). By Proposition 4.1 and (5.6) it follows that for any $\varepsilon>0$ there exists $\delta>0$ such that

$$
\begin{aligned}
\operatorname{dim}_{H}\left(\overline{\mathscr{B}} \cap\left(p_{L}-\delta, p_{L}+\delta\right)\right) & \geq(1-\varepsilon) \operatorname{dim}_{H} \pi_{p_{L}}\left(\mathbf{B}_{\delta}\left(p_{L}\right)\right) \\
& \geq(1-\varepsilon) \operatorname{dim}_{H} \pi_{p_{L}}\left(\Lambda_{N}\right) \\
& =(1-\varepsilon) \frac{h_{t o p}\left(F_{\mathbf{W}_{p_{L}, N}}\left(\alpha_{1} \ldots \alpha_{m}\right)\right)}{\log p_{L}} \\
& =(1-\varepsilon) \frac{h_{t o p}\left(\mathbf{W}_{p_{L}, N}\right)}{\log p_{L}}
\end{aligned}
$$

for all sufficiently large integers $N>N_{1}$. Letting $N \rightarrow \infty$ we conclude by Lemmas 5.3 and 2.4 that

$$
\operatorname{dim}_{H}\left(\overline{\mathscr{B}} \cap\left(p_{L}-\delta, p_{L}+\delta\right)\right) \geq(1-\varepsilon) \frac{h_{t o p}\left(\mathbf{V}_{p_{L}}\right)}{\log p_{L}}=(1-\varepsilon) \operatorname{dim}_{H} \mathcal{U}_{p_{L}} .
$$

Since $\varepsilon>0$ was taken arbitrarily, this establishes the lemma.

Now we prove the lower bound of Theorem 2 for $q \in\left(q_{K L}, q_{T}\right)$ being the left endpoint of an entropy plateau.

Lemma 5.6. Let $\left[p_{L}, p_{R}\right] \subset\left(q_{K L}, q_{T}\right)$ be a plateau of $H$. Then for any $\delta>0$ we have

$$
\operatorname{dim}_{H} \overline{\mathscr{B}} \cap\left(p_{L}-\delta, p_{L}+\delta\right) \geq \operatorname{dim}_{H} \mathcal{U}_{p_{L}}
$$


Proof. The proof is similar to that of Lemma 5.5. We only give the proof for $M=2 k$, since the proof for $M=2 k+1$ is similar.

By Lemma 2.11 (i) it follows that $\alpha\left(p_{L}\right)=\left(\alpha_{i}\right)=\left(\alpha_{1} \ldots \alpha_{m}\right)^{\infty}$ is a $*$-irreducible sequence, where $m$ is the minimal period of $\alpha\left(p_{L}\right)$. Then there exists $n \geq 1$ such that $\xi(n+1) \preccurlyeq \alpha\left(p_{L}\right) \prec$ $\xi(n)$, where $\xi(n)=\lambda_{1} \ldots \lambda_{2^{n-1}}\left(\overline{\lambda_{1} \ldots \lambda_{2^{n-1}}}+\right)^{\infty}$. By (2.4) this implies that $m>2^{n}$. Since $\alpha\left(p_{L}\right)=\left(\alpha_{i}\right)$ is periodic while $\xi(n+1)$ is eventually periodic, we have $\xi(n+1) \prec \alpha\left(p_{L}\right) \prec \xi(n)$. So there exists a large integer $N_{0}$ such that

$$
\xi(n+1) \prec \alpha_{1} \ldots \alpha_{N_{0}} \prec \xi(n) .
$$

Since $\alpha\left(p_{L}\right)=\left(\alpha_{i}\right)$ is $*$-irreducible, by Definition 2.10 there exists an integer $N_{1}>N_{0}$ such that

$$
\alpha_{1} \ldots \alpha_{j}\left({\overline{\alpha_{1} \ldots \alpha_{j}}}^{+}\right)^{\infty} \prec \alpha_{1} \ldots \alpha_{N_{1}} \quad \text { if } \quad\left(\alpha_{1} \ldots \alpha_{j}^{-}\right)^{\infty} \in \mathbf{V} \text { and } 2^{n}<j \leq m .
$$

Let $\nu=\lambda_{1} \ldots \lambda_{2^{n}}^{-}$be the word defined as in Lemma 5.4 (ii). Then by Lemma 2.11 (iii) there exist a large integer $N \geq N_{1}$ and a word $\omega$ such that

$$
\overline{\alpha_{1} \ldots \alpha_{N}} \prec \sigma^{j}\left(\alpha_{1} \ldots \alpha_{m} \omega \nu^{\infty}\right) \prec \alpha_{1} \ldots \alpha_{N} \quad \text { for any } \quad j \geq 0 .
$$

Observe that $\left(\mathbf{W}_{p_{L}, N}, \sigma\right)$ is an $N$-step subshift of finite type. Note by (5.11) that $\alpha_{1} \ldots \alpha_{m} \omega \nu^{N} \in$ $\mathcal{L}\left(\mathbf{W}_{p_{L}, N}\right)$. Then by [30, Theorem 2.1.8] it follows that for any sequence $\left(d_{i}\right) \in F_{\mathbf{W}_{p_{L}, N}}\left(\nu^{N}\right)$ we have $\alpha_{1} \ldots \alpha_{m} \omega d_{1} d_{2} \ldots \in F_{\mathbf{W}_{p_{L}, N}}\left(\alpha_{1} \ldots \alpha_{m}\right)$. This implies

$$
\left\{\alpha_{1} \ldots \alpha_{m} \omega d_{1} d_{2} \ldots:\left(d_{i}\right) \in F_{\mathbf{W}_{p_{L}, N}}\left(\nu^{N}\right)\right\} \subseteq F_{\mathbf{W}_{p_{L}, N}}\left(\alpha_{1} \ldots \alpha_{m}\right) \subseteq \mathbf{W}_{p_{L}, N} .
$$

So, by Lemma 5.4 (ii) we obtain

$$
h_{t o p}\left(F_{\mathbf{W}_{p_{L}, N}}\left(\alpha_{1} \ldots \alpha_{m}\right)\right)=h_{t o p}\left(\mathbf{W}_{p_{L}, N}\right) .
$$

Let $\Delta_{N}$ be the set of sequences $\left(a_{i}\right)$ satisfying

$$
a_{1} \ldots a_{m N}=\left(\alpha_{1} \ldots \alpha_{m}\right)^{N} \quad \text { and } \quad a_{m N+1} a_{m N+2} \ldots \in F_{\mathbf{W}_{p_{L}, N}}\left(\alpha_{1} \ldots \alpha_{m}\right) .
$$

Fix $\delta>0$. Then we claim that

$$
\Delta_{N} \subset \mathbf{B}_{\delta}\left(p_{L}\right)=\left\{\alpha(q): q \in \overline{\mathscr{B}} \cap\left(p_{L}-\delta, p_{L}+\delta\right)\right\}
$$

for all sufficiently large integers $N>N_{1}$. Observe that the common prefix of sequences in $\Delta_{N}$ has length at least $m(N+1)$ and it coincides with a prefix of $\alpha\left(p_{L}\right)=\left(\alpha_{1} \ldots \alpha_{m}\right)^{\infty}$. So, by Lemmas 2.1 and 2.12 it suffices to show that for all integers $N>N_{1}$ any sequence in $\Delta_{N}$ is *-irreducible.

Take $N>N_{1}$ sufficiently large and take $\left(a_{i}\right) \in \Delta_{N}$. Then by (5.9) we have $\xi(n+1) \prec$ $\left(a_{i}\right) \prec \xi(n)$. Furthermore, by Lemma 5.2 and the definition of $\Delta_{N}$ it follows that

$$
\overline{a_{1} \ldots a_{N}} \prec \sigma^{j}\left(\left(a_{i}\right)\right) \prec a_{1} \ldots a_{N} \quad \text { for any } \quad j \geq 1 \text {. }
$$


This implies that $\left(a_{i}\right) \in \mathbf{V}$. Furthermore, by (5.10), (5.13) and arguments similar to those in the proof of Lemma 5.5 we can prove that

$$
a_{1} \ldots a_{j}\left(\overline{a_{1} \ldots a_{j}}+\right)^{\infty} \prec\left(a_{i}\right)
$$

whenever $j>2^{n}$ and $\left(a_{1} \ldots a_{j}^{-}\right)^{\infty} \in \mathbf{V}$. Therefore, by Definition 2.10 the sequence $\left(a_{i}\right)$ is *-irreducible, and then $\Delta_{N} \subset \mathbf{B}_{\delta}\left(p_{L}\right)$ for all $N>N_{1}$, proving the claim.

Hence, by Proposition 4.1 and (5.12) it follows that for any $\varepsilon>0$ there exists $\delta>0$ such that

$$
\begin{aligned}
\operatorname{dim}_{H}\left(\overline{\mathscr{B}} \cap\left(p_{L}-\delta, p_{L}+\delta\right)\right) & \geq(1-\varepsilon) \operatorname{dim}_{H} \pi_{p_{L}}\left(\mathbf{B}_{\delta}\left(p_{L}\right)\right) \\
& \geq(1-\varepsilon) \operatorname{dim}_{H} \pi_{p_{L}}\left(\Delta_{N}\right) \\
& =(1-\varepsilon) \frac{h_{t o p}\left(F_{\mathbf{W}_{p_{L}, N}}\left(\alpha_{1} \ldots \alpha_{m}\right)\right)}{\log p_{L}} \\
& =(1-\varepsilon) \frac{h_{t o p}\left(\mathbf{W}_{p_{L}, N}\right)}{\log p_{L}}
\end{aligned}
$$

for all sufficiently large integers $N>N_{1}$. Letting $N \rightarrow \infty$ we obtain by Lemmas 5.3 and 2.4 that

$$
\operatorname{dim}_{H}\left(\overline{\mathscr{B}} \cap\left(p_{L}-\delta, p_{L}+\delta\right)\right) \geq(1-\varepsilon) \frac{h_{t o p}\left(\mathbf{V}_{p_{L}}\right)}{\log p_{L}}=(1-\varepsilon) \operatorname{dim}_{H} \mathcal{U}_{p_{L}}
$$

Since $\varepsilon>0$ was arbitrary, we complete the proof by letting $\varepsilon \rightarrow 0$.

Proof of Theorem Q Take $q \in \overline{\mathscr{B}}$ and $\delta>0$. By Lemma 2.7 there exists a sequence of plateaus $\left\{\left[p_{L}(n), p_{R}(n)\right]\right\}$ such that $p_{L}(n)$ converges to $q$ as $n \rightarrow \infty$. By Lemmas 5.5 and 5.6 it follows that

$$
\operatorname{dim}_{H}(\overline{\mathscr{B}} \cap(q-\delta, q+\delta)) \geq \operatorname{dim}_{H} \mathcal{U}_{p_{L}(n)}
$$

for all sufficiently large $n$. Letting $n \rightarrow \infty$ and by Lemma 2.4 we obtain that

$$
\operatorname{dim}_{H}(\overline{\mathscr{B}} \cap(q-\delta, q+\delta)) \geq \operatorname{dim}_{H} \mathcal{U}_{q}
$$

Therefore, the theorem follows from (5.14) and Proposition 5.1 .

\section{Dimensional spectrum of $\mathscr{U}$}

Recall that $\mathscr{U}$ is the set of univoque bases $q \in(1, M+1]$ for which 1 has a unique $q$ expansion. In this section we will use Theorem 2 to prove Theorem 3 for the dimensional spectrum of $\mathscr{U}$, which states that

$$
\operatorname{dim}_{H}(\mathscr{U} \cap(1, t])=\max _{q \leq t} \operatorname{dim}_{H} \mathcal{U}_{q} \quad \text { for all } t>1 .
$$

We focus on $t \in\left(q_{K L}, M+1\right)$, since by Lemma 2.4 the other cases are trivial.

Since the proof of Lemma 4.3 above only uses properties of $\overline{\mathscr{U}}$ instead of $\overline{\mathscr{B}}$, the proof also gives the following lemma for the set $\overline{\mathscr{U}}$. 
Lemma 6.1. Let $q \in \overline{\mathscr{U}} \backslash\{M+1\}$. Then for any $\varepsilon>0$ there exists a $\delta>0$ such that

$$
\operatorname{dim}_{H}(\overline{\mathscr{U}} \cap(q-\delta, q+\delta)) \leq(1+\varepsilon) \operatorname{dim}_{H} \pi_{q+\delta}\left(\mathbf{U}_{\delta}(q)\right),
$$

where $\mathbf{U}_{\delta}(q)=\{\alpha(p): p \in \overline{\mathscr{U}} \cap(q-\delta, q+\delta)\}$.

To prove Theorem 3 we first consider the upper bound.

Lemma 6.2. For any $t \in\left(q_{K L}, M+1\right)$ we have

$$
\operatorname{dim}_{H}(\overline{\mathscr{U}} \cap(1, t]) \leq \max _{q \leq t} \operatorname{dim}_{H} \mathcal{U}_{q} .
$$

Proof. Fix $\varepsilon>0$, and take $t \in\left(q_{K L}, M+1\right)$. Then it suffices to prove

$$
\operatorname{dim}_{H}(\overline{\mathscr{U}} \cap(1, t]) \leq(1+\varepsilon)\left(\max _{q \leq t} \operatorname{dim}_{H} \mathcal{U}_{q}+\varepsilon\right) .
$$

By Lemmas 2.4 and 6.1 it follows that for each $q \in \overline{\mathscr{U}} \cap(1, t]$ there exists a sufficiently small $\delta=\delta(q, \varepsilon)>0$ such that

$$
\begin{aligned}
\operatorname{dim}_{H} \mathcal{U}_{q+\delta} & \leq \operatorname{dim}_{H} \mathcal{U}_{q}+\varepsilon \\
\operatorname{dim}_{H}(\overline{\mathscr{U}} \cap(q-\delta, q+\delta)) & \leq(1+\varepsilon) \operatorname{dim}_{H} \pi_{q+\delta}\left(\mathbf{U}_{\delta}(q)\right) .
\end{aligned}
$$

Observe that $\{(q-\delta, q+\delta): q \in \overline{\mathscr{U}} \cap(1, t]\}$ is an open cover of $\overline{\mathscr{U}} \cap(1, t]$, and that $\overline{\mathscr{U}} \cap(1, t]=$ $\overline{\mathscr{U}} \cap\left[q_{K L}, t\right]$ is a compact set. Hence, there exist $q_{1}, q_{2}, \ldots, q_{N}$ in $\overline{\mathscr{U}} \cap(1, t]$ such that

$$
\overline{\mathscr{U}} \cap(1, t] \subseteq \bigcup_{i=1}^{N}\left(\overline{\mathscr{U}} \cap\left(q_{i}-\delta_{i}, q_{i}+\delta_{i}\right)\right),
$$

where $\delta_{i}=\delta\left(q_{i}, \varepsilon\right)$ for $1 \leq i \leq N$.

Note by Lemmas 2.2 and 2.3 that for each $i \in\{1,2, \ldots, N\}$ we have

$$
\pi_{q_{i}+\delta_{i}}\left(\mathbf{U}_{\delta_{i}}\left(q_{i}\right)\right)=\pi_{q_{i}+\delta_{i}}\left(\left\{\alpha(p): p \in \overline{\mathscr{U}} \cap\left(q_{i}-\delta_{i}, q_{i}+\delta_{i}\right)\right\}\right) \subseteq \mathcal{U}_{q_{i}+\delta_{i}} .
$$

Then by (6.2) and (6.3) it follows that

$$
\begin{aligned}
\operatorname{dim}_{H}(\overline{\mathscr{U}} \cap(1, t]) & \leq \operatorname{dim}_{H}\left(\bigcup_{i=1}^{N}\left(\overline{\mathscr{U}} \cap\left(q_{i}-\delta_{i}, q_{i}+\delta_{i}\right)\right)\right) \\
& =\max _{1 \leq i \leq N} \operatorname{dim}_{H}\left(\overline{\mathscr{U}} \cap\left(q_{i}-\delta_{i}, q_{i}+\delta_{i}\right)\right) \\
& \leq(1+\varepsilon) \max _{1 \leq i \leq N} \operatorname{dim}_{H} \pi_{q_{i}+\delta_{i}}\left(\mathbf{U}_{\delta_{i}}\left(q_{i}\right)\right) \\
& \leq(1+\varepsilon) \max _{1 \leq i \leq N} \operatorname{dim}_{H} \mathcal{U}_{q_{i}+\delta_{i}} \\
& \leq(1+\varepsilon) \max _{1 \leq i \leq N}\left(\operatorname{dim}_{H} \mathcal{U}_{q_{i}}+\varepsilon\right) \\
& \leq(1+\varepsilon)\left(\max _{q \leq t} \operatorname{dim}_{H} \mathcal{U}_{q}+\varepsilon\right) .
\end{aligned}
$$

This proves (6.1), and completes the proof. 
The next lemma gives the lower bound of Theorem 3,

Lemma 6.3. For any $t \in\left(q_{K L}, M+1\right)$ we have

$$
\operatorname{dim}_{H}(\overline{\mathscr{U}} \cap(1, t]) \geq \max _{q \leq t} \operatorname{dim}_{H} \mathcal{U}_{q} .
$$

Proof. Take $t \in\left(q_{K L}, M+1\right)$. Note by Lemma 2.4 that the dimension function $D: q \mapsto$ $\operatorname{dim}_{H} \mathcal{U}_{q}$ is continuous. Then there exists $q_{*} \in\left[q_{K L}, t\right]$ such that

$$
\operatorname{dim}_{H} \mathcal{U}_{q_{*}}=\max _{q \leq t} \operatorname{dim}_{H} \mathcal{U}_{q}
$$

Since the entropy function $H$ is locally constant on the complement of $\mathscr{B}$, it follows by Lemma 2.4 that

$$
q_{*} \in\left(q_{K L}, t\right] \backslash \bigcup\left(p_{L}, p_{R}\right] \subseteq\left(q_{K L}, t\right] \cap \overline{\mathscr{B}} .
$$

If $q_{*} \in\left(q_{K L}, t\right) \cap \overline{\mathscr{B}}$, then the lemma follows by $\overline{\mathscr{B}} \subset \overline{\mathscr{U}}$ and Theorem 2 , If $q_{*}=t$, then by Lemma 2.7 (i) there exists a sequence of plateaus $\left\{\left[p_{L}(n), p_{R}(n)\right]\right\}$ such that $p_{L}(n) \in$ $\left(q_{K L}, t\right) \cap \overline{\mathscr{B}}$ and $p_{L}(n) \nearrow q_{*}$ as $n \rightarrow \infty$. Therefore, by Lemma 2.4 and Theorem 2 we also have

$$
\operatorname{dim}_{H}(\overline{\mathscr{U}} \cap(1, t]) \geq \operatorname{dim}_{H}\left(\overline{\mathscr{B}} \cap\left(q_{K L}, t\right]\right) \geq \operatorname{dim}_{H} \mathcal{U}_{p_{L}(n)} \rightarrow \operatorname{dim}_{H} \mathcal{U}_{q_{*}}
$$

as $n \rightarrow \infty$. This establishes the lemma.

Proof of Theorem [3. For $1<t \leq q_{K L}$ we have $\mathscr{U} \cap(1, t] \subseteq\left\{q_{K L}\right\}$ and thus by Lemma 2.4 (i) it follows that

$$
\operatorname{dim}_{H}(\mathscr{U} \cap(1, t])=0=\max _{q \leq t} \operatorname{dim}_{H} \mathcal{U}_{q} .
$$

For $t \geq M+1$ we have $\mathscr{U}=\mathscr{U} \cap(1, t]$ and the result also follows from Lemma 2.4, For the remaining $t$ the result follows from Lemmas 6.2 and 6.3, since $\overline{\mathscr{U}} \backslash \mathscr{U}$ is countable.

From Lemma 2.4 it follows that the dimension function $D: q \mapsto \operatorname{dim}_{H} \mathcal{U}_{q}$ has a Devil's staircase behavior (see also Remark 2.5 (1)). This implies that $\phi(t):=\max _{q \leq t} \operatorname{dim}_{H} \mathcal{U}_{q}$ is a Devil's staircase in $(1, \infty)$ : (i) $\phi$ is non-decreasing and continuous in $(1, \infty)$; (ii) $\phi$ is locally constant almost everywhere in $(1, \infty)$; and (iii) $\phi\left(q_{K L}\right)=0$, and $\phi(t)>0$ for any $t>q_{K L}$.

\section{VARIATIONS OF $\mathscr{U}(M)$}

For any $K \in\{0,1, \ldots, M\}$, let $\mathscr{U}(K)$ denote the set of bases $q>1$ such that 1 has a unique $q$-expansion over the alphabet $\{0,1, \ldots, K\}$. Then $\mathscr{U}(K) \subset(1, K+1]$. In this section we investigate the Hausdorff dimension of the intersection $\bigcap_{J=K}^{M} \mathscr{U}(J)$, and prove Theorem 4 . Note that $q_{K L}=q_{K L}(M)$ is the smallest element of $\mathscr{U}(M)$, and $K+1$ is the largest element of $\mathscr{U}(K)$. So, if $K+1<q_{K L}$ then $\mathscr{U}(M) \cap \mathscr{U}(K)=\emptyset$. Therefore, in the following we assume $K \in\left[q_{K L}-1, M\right]$. 
Lemma 7.1. Let $K \in\left[q_{K L}-1, M\right]$ be an integer. Then for each $q \in \mathscr{U}(M) \cap(1, K+1]$ the unique expansion $\alpha(q)=\left(\alpha_{i}(q)\right)$ satisfies

$$
M-K \leq \alpha_{i}(q) \leq K \quad \text { for any } \quad i \geq 1
$$

Proof. Clearly, the lemma holds if $K=M$. So we assume $K<M$. Take $q \in \mathscr{U}(M) \cap(1, K+$ 1] $\subseteq\left[q_{K L}, K+1\right]$. Then

$$
\alpha\left(q_{K L}\right) \preceq \alpha(q) \preceq \alpha(K+1)=K^{\infty} .
$$

This, together with $\alpha_{1}\left(q_{K L}\right) \geq M-\alpha_{1}\left(q_{K L}\right)$, implies that

$$
M-K \leq \alpha_{1}\left(q_{K L}\right) \leq \alpha_{1}(q) \leq K
$$

Since $M>K$ and $q \in \mathscr{U}(M)$, it follows from Lemma 2.3 (i) that

$$
M-K \leq M-\alpha_{1}(q) \leq \alpha_{i}(q) \leq \alpha_{1}(q) \leq K \quad \text { for any } \quad i \geq 1
$$

This completes the proof.

Lemma 7.2. Let $K \in\left[q_{K L}-1, M\right]$ be an integer. Then

$$
\mathscr{U}(M) \cap \mathscr{U}(K)=(1, K+1] \cap \mathscr{U}(M) .
$$

Proof. Since $\mathscr{U}(K) \subseteq(1, K+1]$, it suffices to prove that $\mathscr{U}(M) \cap(1, K+1] \subseteq \mathscr{U}(K)$. Take $q \in \mathscr{U}(M) \cap(1, K+1]$. Then by Lemma 2.3 it follows that $\alpha(q)=\left(\alpha_{i}(q)\right)$ satisfies

$$
\left(K-\alpha_{i}(q)\right) \preceq\left(M-\alpha_{i}(q)\right) \prec \alpha_{i+1}(q) \alpha_{i+2}(q) \cdots \prec \alpha(q) \text { for all } i \geq 1
$$

Note by Lemma 7.1 that $0 \leq \alpha_{i}(q) \leq K$ for all $i \geq 1$. Hence, by (7.1) and Lemma 2.3 we conclude that $q \in \mathscr{U}(K)$.

Proof of Theorem 4. First we prove (i). Clearly, if $K<q_{K L}-1$ then $\bigcap_{J=K}^{M} \mathscr{U}(J)=\emptyset$, and therefore (i) holds by Lemma 2.4 (i). If $q_{K L}-1 \leq K \leq M$, then by repeatedly using Lemma 
7.2 we conclude that

$$
\begin{aligned}
\bigcap_{J=K}^{M} \mathscr{U}(J)= & (\mathscr{U}(M) \cap \mathscr{U}(M-1)) \cap \bigcap_{J=K}^{M-2} \mathscr{U}(J) \\
= & (1, M] \cap \mathscr{U}(M) \cap \bigcap_{J=K}^{M-2} \mathscr{U}(J) \\
= & (1, M] \cap(\mathscr{U}(M) \cap \mathscr{U}(M-2)) \cap \bigcap_{J=K}^{M-3} \mathscr{U}(J) \\
= & (1, M-1] \cap \mathscr{U}(M) \cap \bigcap_{J=K}^{M-3} \mathscr{U}(J) \\
& \ldots \\
= & (1, K+1] \cap \mathscr{U}(M) .
\end{aligned}
$$

Therefore, by Theorem 3 we establish (i).

As for (ii), we observe that for any $L \geq 1$,

$$
\mathscr{U}(L)=\left(\mathscr{U}(L) \backslash \bigcup_{J \neq L} \mathscr{U}(J)\right) \cup \bigcup_{J \neq L}(\mathscr{U}(L) \cap \mathscr{U}(J)) .
$$

From (i) and Lemma 2.4 (i) it follows that $\operatorname{dim}_{H}(\mathscr{U}(L) \cap \mathscr{U}(J))<1$ for any $J \neq L$. Furthermore, by Lemma 2.6 we have $\operatorname{dim}_{H} \mathscr{U}(L)=1$ (see also, [23, Theorem 1.6]). Therefore, (ii) immediately follows from (7.2).

\section{FinAL REMARKS}

It was shown in Theorem 3 that the function $\phi(t)=\operatorname{dim}_{H}(\mathscr{U} \cap(1, t])$ is a Devil's staircase in $(1, \infty)$ (see Figure 1 for the sketch plot of $\phi$ ). Then a natural question is to ask about the presence and position of plateaus for $\phi$, i.e., maximal intervals on which $\phi$ is constant. By Lemma 2.4 (i) and Theorem 3 it follows that $\phi(t)=0$ if and only if $t \leq q_{K L}$, and $\phi(t)=1$ if and only if $t \geq M+1$. Hence, the first plateau of $\phi$ is $\left(1, q_{K L}\right]$, and the last plateau is $[M+1, \infty)$.

Since $\phi(t)=\max _{q \leq t} \operatorname{dim}_{H} \mathcal{U}_{q}$, an interval $\left[q_{L}, q_{R}\right]$ is a plateau of $\phi$ if and only if

$$
\begin{array}{llr}
\operatorname{dim}_{H} \mathcal{U}_{p}<\operatorname{dim}_{H} \mathcal{U}_{q_{L}} & \text { for any } & p<q_{L}, \\
\operatorname{dim}_{H} \mathcal{U}_{q} \leq \operatorname{dim}_{H} \mathcal{U}_{q_{L}} & \text { for any } & q_{L} \leq q \leq q_{R}, \\
\operatorname{dim}_{H} \mathcal{U}_{r}>\operatorname{dim}_{H} \mathcal{U}_{q_{L}} & \text { for any } & r>q_{R}
\end{array}
$$

By Lemma 2.4 it follows that for each plateau $\left[q_{L}, q_{R}\right]$ of $\phi$ we have $\operatorname{dim}_{H} \mathcal{U}_{q_{L}}=\operatorname{dim}_{H} \mathcal{U}_{q_{R}}$.

Question 1. Can we describe the plateaus of $\phi$ in $\left(q_{K L}, M+1\right)$ ? 
Theorem 3 tells us that the set $\mathscr{U}$ gets heavier towards the right, but does not say anything about the local weight.

Question 2. For any $t_{2}>t_{1}>1$, what is the local dimension $\operatorname{dim}_{H}\left(\mathscr{U} \cap\left[t_{1}, t_{2}\right]\right)$ ?

Acknowledgements. The authors thank the anonymous referee for many useful suggestions. The second author was supported by NSFC No. 11401516. The third author was supported by NSFC No. 11671147, 11571144 and Science and Technology Commission of Shanghai Municipality (STCSM) No. 18dz2271000. The forth author was supported by NSFC No. 11601358.

\section{REFERENCES}

[1] R. Alcaraz Barrera, S. Baker, and D. Kong, Entropy, topological transitivity, and dimensional properties of unique q-expansions, arXiv:1609.02122v2 (2018), to appear in Trans. Amer. Math. Soc.

[2] P. C. Allaart, S. Baker, and D. Kong. Bifurcation sets arising from non-integer base expansions, arXiv:1706.05190 (2018), to appear in Journal of Fractal Geometry.

[3] P. C. Allaart and D. Kong. On the continuity of the Hausdorff dimension of the univoque set, arXiv:1804.02879 (2018).

[4] J.-P. Allouche, Théorie des nombres et automates, Thèse d'État, Université Bordeaux I (1983).

[5] J.-P. Allouche and J. Shallit, The ubiquitous Prouhet-Thue-Morse sequence, Sequences and their applications (Singapore, 1998), 1-16, Springer Ser. Discrete Math. Theor. Comput. Sci., Springer, London (1999).

[6] J.-P. Allouche and M. Cosnard, Itérations de fonctions unimodales et suites engendrées pas automates, C. R. Acad. Sci. Paris Sr. I Math. 296 (1983), 159-162.

[7] J.-P. Allouche and M. Cosnard, Non-integer bases, iteration of continuous real maps, and an arithmetic self-similar set, Acta Math. Hungar. 91 (2001), 325-332.

[8] C. Baiocchi and V. Komornik, Greedy and quasi-greedy expansions in non-integer bases, arXiv:0710.3001v1 (2007).

[9] C. Bonanno, C. Carminati, S. Isola and G. Tiozzo, Dynamics of Continued Fractions and Kneading Sequences of Unimodal Maps, Discrete Contin. Dyn. Syst. 33 (2013), 1313-1332.

[10] C. Carminati and G. Tiozzo, A canonical thickening of $\mathbb{Q}$ and the entropy of $\alpha$-continued fraction transformations, Ergodic Theory Dynam. Systems 32 (2012), 1249-1269.

[11] K. Dajani and M. de Vries, Invariant densities for random $\beta$-expansions, J. Eur. Math. Soc. 9 (2007), $157-176$.

[12] K. Dajani and C. Kalle, Invariant measures, matching and the frequency of 0 for signed binary expansions, arXiv:1703.06335 (2017).

[13] Z. Daróczy and I. Kátai, Univoque sequences, Publ. Math. Debrecen 42 (1993), 397-407. 
[14] Z. Daróczy and I. Kátai, On the structure of univoque numbers, Publ. Math. Debrecen 46 (1995), 385-408.

[15] M. de Vries and V. Komornik, Unique expansions of real numbers, Adv. Math. 221 (2009), 390-427.

[16] M. de Vries and V. Komornik, Expansions in non-integer bases, Combinatorics, words and symbolic dynamics, Encyclopedia Math. Appl., vol. 159 (Cambridge Univ. Press, Cambridge, pp. 18-58, 2016).

[17] M. de Vries, V. Komornik, and P. Loreti, Topology of the set of univoque bases, Topology Appl. 205 (2016), 117-137.

[18] P. Erdős, I. Joó, and V. Komornik, Characterization of the unique expansions $1=\sum_{i=1}^{\infty} q^{-n_{i}}$ and related problems, Bull. Soc. Math. France 118 (1990), 377-390.

[19] P. Erdös and I. Joó, On the number of expansions $1=\sum q^{-n_{i}}$, Ann. Univ. Sci. Budapest. Eötvös Sect. Math. 35 (1992), 129-132.

[20] K. Falconer, Fractal geometry: Mathematical foundations and applications, John Wiley \& Sons Ltd., Chichester, 1990.

[21] P. Glendinning and N. Sidorov, Unique representations of real numbers in non-integer bases, Math. Res. Lett. 8 (2001), 535-543.

[22] V. Komornik, Expansions in noninteger bases, Integers 11B, Paper No. A9, 30 (2011).

[23] V. Komornik, D. Kong, and W. Li, Hausdorff dimension of univoque sets and devil's staircase, Adv. Math. 305 (2017), 165-196.

[24] V. Komornik and P. Loreti, Subexpansions, superexpansions and uniqueness properties in non-integer bases, Period. Math. Hungar. 44 (2002), 197-218.

[25] V. Komornik and P. Loreti, On the topological structure of univoque sets, J. Number Theory 122 (2007), 157-183.

[26] D. Kong and W. Li, Hausdorff dimension of unique beta expansions, Nonlinearity 28 (2015), 187-209.

[27] D. Kong, W. Li, and M. Dekking, Intersections of homogeneous Cantor sets and beta-expansions, Nonlinearity 23 (2010), 2815-2834.

[28] D. Kong, W. Li, F. Lü, and M. de Vries, Univoque bases and Hausdorff dimensions, Monatsh. Math. 184 (2017), 443-458.

[29] C. Kraaikamp, T. Schmidt and W. Steiner, Natural extensions and entropy of $\alpha$-continued fractions, Nonlinearity 25 (2012), 2207-2243.

[30] D. Lind and B. Marcus, An introduction to symbolic dynamics and coding, Cambridge University Press, Cambridge, 1995.

[31] R. D. Mauldin and S. C. Williams, Hausdorff dimension in graph directed constructions, Trans. Amer. Math. Soc. 309 (1988), 811-829.

[32] H. Nakada, Metrical theory for a class of continued fraction transformations and their natural extensions, Tokyo J. Math. 4 (1981), 399-426.

[33] W. Parry, On the $\beta$-expansions of real numbers, Acta Math. Acad. Sci. Hungar. 11 (1960), 401-416. 
[34] A. Rényi, Representations for real numbers and their ergodic properties, Acta Math. Acad. Sci. Hungar. 8 (1957), 477-493.

[35] N. Sidorov, Almost every number has a continuum of $\beta$-expansions, Amer. Math. Monthly 110 (2003), 838-842.

[36] N. Sidorov, Arithmetic dynamics, Topics in dynamics and ergodic theory, London Math. Soc. Lecture Note Ser., vol. 310, Cambridge Univ. Press, Cambridge, pp. 145-189, 2003.

[37] N. Sidorov, Expansions in non-integer bases: lower, middle and top orders, J. Number Theory 129 (2009), 741-754.

(C. Kalle) Mathematical Institute, University of Leiden, PO Box 9512, 2300 RA Leiden, The Netherlands

E-mail address: kallecccj@math.leidenuniv.nl

(D. Kong) Mathematical Institute, University of Leiden, PO Box 9512, 2300 RA Leiden, The Netherlands

Current address: College of Mathematics and Statistics, Chongqing University, 401331 Chongqing, China E-mail address, Corresponding author: derongkong@126.com

(W. Li) School of Mathematical Sciences, Shanghai Key Laboratory of PMmp, East China Normal University, Shanghai 200062, People's Republic of China

E-mail address: wxli@math.ecnu.edu.cn

(F. Lü) Department of Mathematics, Sichuan Normal University, Chengdu 610068, People's RePublic OF China

E-mail address: lvfan1123@163.com 